\title{
Systematic Review \\ Effect of High-Intensity Interval Training on Physical Health in Coronary Artery Disease Patients: A Meta-Analysis of Randomized Controlled Trials
}

\author{
Litao Du ${ }^{1,+}$, Xianliang Zhang ${ }^{1,+}$, Ke Chen ${ }^{1}$, Xiaoyu Ren ${ }^{1}$, Si Chen ${ }^{2, *}$ and Qiang He ${ }^{1, *}$ (i) \\ 1 School of Physical Education, Shandong University, Jinan 250012, China; tao7221@oulook.com (L.D.); \\ xlzhang@sdu.edu.cn (X.Z.); chenke20210813@163.com (K.C.); rxyd163yx@163.com (X.R.) \\ 2 School of Nursing and Rehabilitation, Cheeloo College of Medicine, Shandong University, Jinan 250102, China \\ * Correspondence: chensi@sdu.edu.cn (S.C.); hq@sdu.edu.cn (Q.H.); Tel.: +86-0531-8838-2000 (S.C.); \\ +86-0531-8839-6626 (Q.H.) \\ + These authors contributed equally to this work.
}

check for updates

Citation: Du, L.; Zhang, X.; Chen, K.; Ren, X.; Chen, S.; He, Q. Effect of High-Intensity Interval Training on Physical Health in Coronary Artery Disease Patients: A Meta-Analysis of Randomized Controlled Trials. J. Cardiovasc. Dev. Dis. 2021, 8, 158. https://doi.org/10.3390/jcdd8110158

Academic Editor: Monique R. M. Jongbloed

Received: 11 September 2021 Accepted: 15 November 2021 Published: 18 November 2021

Publisher's Note: MDPI stays neutral with regard to jurisdictional claims in published maps and institutional affiliations.

Copyright: (c) 2021 by the authors. Licensee MDPI, Basel, Switzerland. This article is an open access article distributed under the terms and conditions of the Creative Commons Attribution (CC BY) license (https:/ / creativecommons.org/licenses/by/ $4.0 /)$.

\begin{abstract}
The effects of exercise-based cardiac rehabilitation (CR) on physical health in coronary artery disease (CAD) patients has long been established, while the optimal exercise mode remains to be determined. This meta-analysis compared the efficacy of high-intensity interval training (HIIT) versus moderate-intensity continuous training (MICT) in CAD patients. Databases were searched up to December 2020. Twenty-five studies with 1272 participants were analyzed. The results showed that both HIIT and MICT induced significant $\mathrm{VO}_{2 \text { peak }}$ improvement with a $4.52 \mathrm{~mL} / \mathrm{kg} / \mathrm{min}$ $(p<0.01)$ and $2.36 \mathrm{~mL} / \mathrm{kg} / \mathrm{min}(p<0.01)$, respectively. Additionally, a larger improvement of $\mathrm{VO}_{2 \text { peak }}(1.92 \mathrm{~mL} / \mathrm{kg} / \mathrm{min}, p<0.01)$ was observed in HIIT over MICT. HIIT with medium and long intervals, higher work/rest ratio induced larger $\mathrm{VO}_{2 \text { peak }}$ improvement than the compared subgroup. Interestingly, non-isocaloric exercise protocols induced larger $\mathrm{VO}_{2 \text { peak }}$ improvement compared with isocaloric protocols. In addition, both HIIT and MICT significantly increased anaerobic threshold and peak power with HIIT superior to MICT. No significant different changes were observed in blood pressure after HIIT or MICT intervention, however when HIIT was compared with MICT, MICT seems superior to HIIT in reducing systolic blood pressure $(-3.61 \mathrm{mmHg}, p<0.01)$ and diastolic blood pressure $(-2.37 \mathrm{mmHg}, p<0.01)$. Although, HIIT and MICT induced significant improvement of most other parameters, like $\mathrm{HR}_{\text {rest }}, \mathrm{HR}_{\text {peak }}$, left ventricular ejection fraction (LVEF), quality of life (QoL), no significant differences were noted between groups. This meta-analysis suggested that HIIT is superior to MICT in increasing $\mathrm{VO}_{2 \text { peak, }}$, anaerobic threshold, peak power in CAD patients. Additionally, the efficacy of HIIT over MICT in improving $\mathrm{VO}_{2 \text { peaks }}$ was influenced by HIIT intervals, work/rest ratio and total caloric consumption. Both HIIT and MICT did not significantly influence resting BP, however, MICT seemed to be more effective in reducing BP than HIIT. HIIT and MICT equally significantly influenced $\mathrm{HR}_{\text {rest }}, \mathrm{HR}_{\text {peak }}$, $\mathrm{HRR} 1 \mathrm{~min}$, OUES, LVEF\%, QoL.
\end{abstract}

Keywords: high-intensity interval training; moderate-intensity continuous training; coronary artery disease; exercise-based cardiac rehabilitation

\section{Introduction}

Cardiovascular diseases are the leading cause of death worldwide. The prevalence and mortality of cardiovascular diseases are currently still on the rise around the world. In China, it is estimated that 11.4 million people live with coronary artery disease (CAD) according to a report on cardiovascular health and disease issued in 2020 by the National Center for Cardiovascular Disease [1]. Regular aerobic exercise training induces positive adaptions to heart and vasculature in patients with $\mathrm{CAD}$, contributing to the improvement of peak oxygen uptake $\left(\mathrm{VO}_{2 \text { peak }}\right)$, which has been established as a strong predictor of cardiovascular [2] and all-cause mortality [3]. Exercise-based CR is also beneficial for 
improving various cardiovascular risk factors, including blood pressure [4], blood lipids [5], insulin dynamics [4], and increasing health-related quality of life(QoL) [6]. Despite the widely established benefits of exercise-based CR on CAD, the most efficient exercise modality has yet to be determined [7]. In early years, the American College of Sports Medicine (ACSM) and American heart association (AHA) recommended an intensity of $50-90 \%$ peak heart rate $\left(\mathrm{HR}_{\text {peak }}\right)$ or $45-85 \% \mathrm{VO}_{2 \text { peak }}$, or $45-85 \%$ heart rate reserve (HRR), which corresponded to 12-16 on the Borg Scale of 6-20 to those with cardiovascular diseases [8].

Exercise-based CR mainly involves MICT, similar to running, walking, or cycling for a long period of time (30-60 min) under moderate intensity (40-80\% $\mathrm{VO}_{2 \text { peak }}$ [9]. MICT is sufficient to reduce cardiovascular risk and mortality [10] and for many years it has been regarded as the essential program adopted in exercise-based CR for stable CAD patients [11]. However, the compliance of exercise-based CR is still low, which might be associated with the enjoyment of the exercise protocol. Recent studies reported similar or better enjoyment and adherence levels by HIIT compared with MICT in healthy or obese adults [12,13]. Additionally, many studies have reported that HIIT, which consists of low volume intermittent high-intensity working (85-100\% $\mathrm{VO}_{2 \text { peak }}$ ) interspersed by active recovery periods, may be a more effective intervention on aerobic capacity, blood pressure, body composition, and QoL in CAD patients, and several narrative reviews have confirmed the positive effects of HIIT in CR $[14,15]$. Several systematic reviews have compared the effectiveness of HIIT and MICT in CAD patients. All these studies investigated the changes of $\mathrm{VO}_{2 \text { peak }}$ in HIIT when compared with MICT. However, limited by a few numbers of included studies [16] or a mixture of heart failure in CAD patients [17-19], the conclusion was constrained with high heterogeneity. In addition, regarding health outcomes such as other cardiorespiratory parameters, cardiovascular risk factors, left ventricular function and quality of life, there are a lack of investigations and the existing results remain inconsistent. Moreover, a great number of relevant RCTs were published in the last three or four years, which have not yet been analyzed in the latest systematic review. A comprehensive review of the relevant literature is needed to resolve these limitations and determine the efficacy of HIIT vs. MICT in CAD patients. This would be helpful to develop a more targeted and efficient exercise prescription and contribute to more alterative choices in CR management.

Therefore, this systematic review and meta-analysis included the latest RCTs aims to evaluate the broad-spectrum physical health benefits of HIIT compared with MICT, with a specific focus on cardiorespiratory fitness, heart rate, blood pressure, blood lipids, left ventricular function and QoL in CAD patients without reduced LVEF or heart failure.

\section{Materials and Methods}

This review was conducted in accordance with the guidelines from the Preferred Reporting for Systematic Review and Meta-Analysis (PROSPERO CRD42021221248) [20].

\subsection{Search Strategy and Selection Criteria}

A systematic literature search of RCTs was conducted in PubMed, Web of Science, SPORTDiscus, Cochrane Library and CNKI up to December 2020. The search was performed using two blocks of terms (e.g., high-intensity interval training, aerobic interval training) and CAD (e.g., coronary artery disease or myocardial infarction) (Table S1). Moreover, reference lists of retrieved articles were hand searched for trials which may meet inclusion criteria but cannot be retrieved in the initial searching. The literature search was performed independently by two reviewers (L.D. and K.C.). Irrelevant studies and duplicates were removed, and then titles and abstracts were fully screened. Any disagreement between the reviewers for inclusion was resolved by the senior authors (S.C., X.Z. and Q.H.). 


\subsection{Selection Criteria and Outcome Measure}

Studies were considered to be eligible for inclusion according to the following criteria: (1) RCTs compared the effectiveness of HIIT(e.g., $\geq 85 \% \mathrm{VO}_{2}$ peak or $\geq 85 \%$ heart-rate reserve $[\mathrm{HRR}]$ or $\geq 90 \%$ heart-rate max [HRM] or equivalent) with $\mathrm{MICT}\left(50-75 \% \mathrm{VO}_{2 \text { peak }}\right.$ or $50-75 \%$ HRR or $50-80 \%$ HRM or equivalent) [21] in participants with CAD without impaired LVEF. (2) Intervention duration lasted for at least 4 weeks. (3) At least one of the following outcomes were measured: $\mathrm{VO}_{2 \text { peak }}$, peak $\mathrm{O}_{2}$ pulse, anaerobic threshold (AT), the ventilatory efficiency slope $\left(\mathrm{VE} / \mathrm{VCO}_{2}\right)$, oxygen uptake efficiency slope (OUES), respiratory exchange ratio (RER), peak power, peak heart rate $\left(\mathrm{HR}_{\text {peak }}\right)$, resting heart rate $\left(\mathrm{HR}_{\text {rest }}\right)$, hear rate recovery at $1 \mathrm{~min}$ (HRR1min), total Cholesterol(TC), high-density lipoprotein cholesterol (HDL-C), low density lipoprotein cholesterol (LDL-C), triglycerides(TG), fasting blood glucose (FBG), resting systolic blood pressure (SBP), resting diastolic blood pressure (DBP), QoL, LVEF, left ventricular end-diastolic diameter (LVEDD), left ventricular endsystolic diameter (LVESD), left ventricular end-diastolic volume(LVEDV), left ventricular end-systolic volume (LVESV). (4) Written in English or Chinese. The exclusion included any study not meeting any of the criteria listed above as follows: (1) non-randomized or uncontrolled, cross-sectional studies; (2) unpublished documents, dissertations, or conference papers.

\subsection{Data Extraction}

Data were extracted by two reviewers (L.D. and K.C.) independently using a standardized form and checked by the third reviewer (X.R.). The collected information included participant information (age, sex, sample size, disease status); characteristics of intervention (intensity, work/rest ratio, duration, and frequency, duration of the intervention, dropout rates); outcome measures (pre- and postintervention means, standard deviation).

\subsection{Risk of Bias and Quality Assessment}

The Cochrane risk-of-bias tool for randomized trials (RoB 2, 2018 beta version), which is structured into five domains including randomization process, deviations from intended interventions, missing outcome data, measurement of the outcome and selection of the reported result, was used to assess the quality of included studies independently by two reviewers (L.D. and K.C.). The disagreement was discussed and resolved by consensus and consultation with the expert group (X.Z., S.C. and Q.H.).

\subsection{Statistical Analyses}

A random-effects model was conducted to determine the pooled effect size of HIIT and MICT on physical health benefits, using Review Manager (RevMan, Version 5.4. The Cochrane Collaboration, Copenhagen, Denmark) to calculate the weight mean difference (WMD) or standardized mean difference (SMD). The significance level of overall effects was set at $p<0.05$. Statistical heterogeneity was assessed using the $I^{2}$ statistic with alpha value for statistical significance of 0.10 indicating significant heterogeneity. Heterogeneity estimates of $25 \%, 50 \%$ and $75 \%$ were considered to be low, moderate, and high heterogeneity, respectively. In order to examine the influence of different training characteristics, a subgroup analysis was performed on $\mathrm{VO}_{2 \text { peak }}$ according to whole intervention duration ( $<12$ weeks, $\geq 12$ weeks), exercise mode (treadmill, cycle ergometer), durations of HIIT interval ( $\leq 1 \mathrm{~min}, 1-3 \mathrm{~min}, \geq 4 \mathrm{~min})$, work / rest ratio of HIIT $(<1, \geq 1)$ and energy consumption (isocaloric, isocaloric). Fixed-effects models were used to compare the subgroups. Multiple sensitivity analyses were performed to determine if any of the results were influenced by the studies that were removed. The funnel plot and the Egger test were used to examine publication bias. 


\section{Results}

\subsection{Study Identification and Selection}

A total of 1550 potentially relevant studies were retrieved initially. After removing duplicates and records not meeting the inclusion criteria by abstract screening, 65 studies were full text reviewed for eligibility. Several studies performed by the same intervention trail produced more than one publication [22-30]. If these papers repeatedly reported the same outcome, such as $\mathrm{VO}_{2 \text { peak, }}$, which was the only outcome evaluated in the metaanalysis, these papers would not be repeatedly included except the one firstly published. However, if $\mathrm{VO}_{2 \text { peak }}$ was not the only outcome assessed, as with our manuscript, and these papers reported other outcomes related to our study, they were included $[23,25,26,29]$. After searching reference lists of retrieved articles for trials which may meet the inclusion criteria of our analysis, 25 articles were eventually included. The flowchart of the whole literature retrieval process is shown in Figure 1.
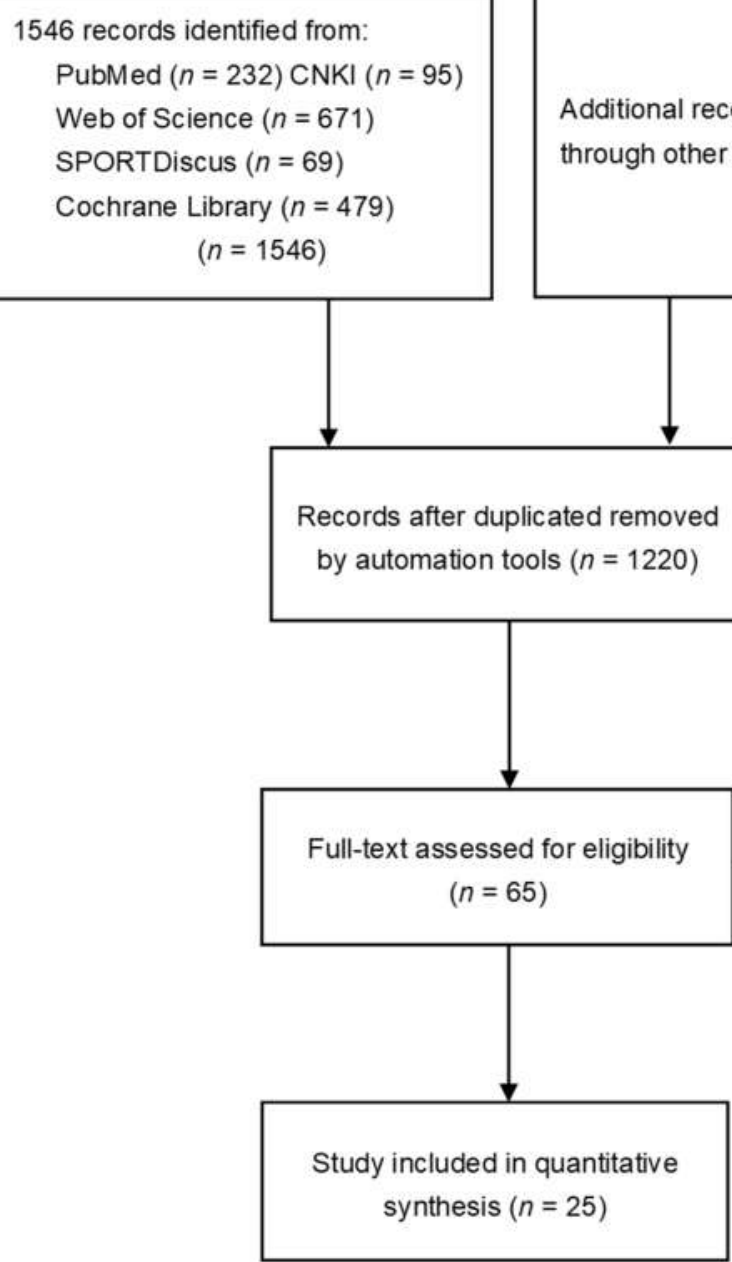

Figure 1. PRISMA flow chart.

\subsection{Characteristics of the Studies}

The characteristics of included studies were shown in Table 1. More than $40 \%$ of all analyzed patients were from Europe [22,23,25,26,28-33]. Eight trials were conducted in America [27,34-40] and five in Asia [41-45]. One trial was conducted in Australia [46] and Egypt [47], respectively. A total of 1272 participants (HIIT 621, MICT 651) were analyzed with a dropout rate ranging from $0 \%$ to $38 \%$ in HIIT and $0 \%$ to $28 \%$ in MICT. The main reason for dropout was low compliance with exercise protocols or withdrawal of consent, rather than medical reasons. A total of six patients failed to complete the study because of 
angina, atrial fibrillation, pericarditis, or myocardial infarction. Most of the trials enrolled both male and female patients except four, which only recruited males [35,36,45]. A total of 1002 males and 201 females were included in this meta-analysis, while Eser et al. 2020 enrolled 69 CAD patients without reporting gender details [31]. The intervention details of the included studies could be found in Table S2. Exercise duration ranged from $4[32,46]$ to $16[34,39]$ weeks with a frequency of 2-3 days a week in most studies except for one study which performed training for 5 days a week [32]. Treadmill running and ergometer cycling were the main exercise protocols.

Table 1. Study characteristics.

\begin{tabular}{|c|c|c|c|c|}
\hline Study & Disease & Subjects $(N, \mathrm{DR})$ & Ages $(M \pm S D)$ & Outcomes \\
\hline Abdelhalem 2018, Egypt & CAD & $\begin{array}{c}\text { HIIT }(18 \mathrm{M} / 2 \mathrm{~F}) \\
\mathrm{MICT}(16 \mathrm{M} / 4 \mathrm{~F}), \mathrm{ND}\end{array}$ & $\begin{array}{l}\text { HIIT }(54.65 \pm 7.63) \\
\text { MICT }(51.95 \pm 8.07)\end{array}$ & LVEF, TC, LDL-C, HDL-C, TG \\
\hline $\begin{array}{l}\text { Amundsen 2008, Norway } \\
\text { Rognmo 2004, Norway }\end{array}$ & CAD & $\begin{array}{l}\text { HIIT (6M/2F, 27\%) } \\
\operatorname{MICT}(8 \mathrm{M} / 1 \mathrm{~F}, 10 \%)\end{array}$ & $\begin{array}{l}\text { HIIT }(63 \pm 11) \\
\operatorname{MICT}(61 \pm 7)\end{array}$ & $\begin{array}{c}\text { LVEDD } \\
\mathrm{VO}_{2 \text { peak }}, \mathrm{SBP}, \mathrm{DBP}, \mathrm{RER}, \mathrm{HR}_{\text {rest }}, \mathrm{HR}_{\text {peak }}\end{array}$ \\
\hline Cardozo 2015, Brazil & CAD & $\begin{array}{c}\text { HIIT }(15 \mathrm{M} / 5 \mathrm{~F}) \\
\mathrm{MICT}(16 \mathrm{M} / 8 \mathrm{~F}), \mathrm{ND}\end{array}$ & $\begin{array}{l}\text { HIIT }(56 \pm 12) \\
\text { MICT }(62 \pm 12)\end{array}$ & $\begin{array}{c}\mathrm{VO}_{2 \text { peak }}, \mathrm{AT} \text {, peak } \mathrm{O}_{2} \text { pulse, } \mathrm{VE} / \mathrm{VCO}_{2} \\
\text { OUES, RER, } \mathrm{HR}_{\text {peak }}\end{array}$ \\
\hline Choi 2018, South Korea & MI & $\begin{array}{l}\text { HIIT (21M/2F, 4\%) } \\
\text { MICT }(18 \mathrm{M} / 3 \mathrm{~F}, 5 \%)\end{array}$ & $\begin{array}{c}\text { HIIT }(53 \pm 6.84) \\
\text { MICT }(57.31 \pm 12.62)\end{array}$ & $\mathrm{VO}_{2 \text { peak }}$ \\
\hline $\begin{array}{c}\text { Conraads 2015, Belgium } \\
\text { Pattyn 2017, Belgium Van } \\
\text { De Heyning 2018, } \\
\text { Belgium }\end{array}$ & CAD & $\begin{array}{l}\text { HIIT }(81 \mathrm{M} / 4 \mathrm{~F}, 15 \%) \\
\operatorname{MICT}(80 \mathrm{M} / 9 \mathrm{~F}, 11 \%)\end{array}$ & $\begin{array}{c}\operatorname{HIIT}(57 \pm 8.8) \\
\operatorname{MICT}(59.9 \pm 9.2)\end{array}$ & $\begin{array}{c}\text { SBP, DBP, QoL, FBG, TC, LDL-C, } \\
\text { HDL-C, TG, HR } \mathrm{Heak}_{\text {peak power, }} \text { Peak } \\
\text { HR }_{\text {rest }}, \text { RER, peak } \mathrm{O}_{2} \text { pulse, } \mathrm{VO}_{2 \text { peak, }} \\
\text { AT, HRR1min, } \\
\text { OUES, VE/VCO }{ }_{2} \text { LVEDD, LVEDV, } \\
\text { LVESD, LVESV }\end{array}$ \\
\hline Currie 2013A, Canada & CAD & $\begin{array}{c}\text { HIIT (7M) } \\
\text { MICT (7M), Total 39\% }\end{array}$ & $\begin{array}{l}\text { HIIT }(63 \pm 11) \\
\operatorname{MICT}(64 \pm 6)\end{array}$ & $\begin{array}{c}\mathrm{VO}_{2 \text { peak }}, \mathrm{AT} \text {, peak power, SBP, DBP } \\
\mathrm{HR}_{\text {peak }}, \mathrm{HR}_{\text {rest }}, \mathrm{RER}\end{array}$ \\
\hline Currie 2013B, Canada & CAD & $\begin{array}{l}\text { HIIT }(10 \mathrm{M} / 1 \mathrm{~F}) \\
\text { MICT }(10 \mathrm{M} / 1 \mathrm{~F}) \\
\text { Total } 27 \%\end{array}$ & $\begin{array}{l}\text { HIIT }(62 \pm 11) \\
\operatorname{MICT}(68 \pm 8)\end{array}$ & $\begin{array}{l}\mathrm{VO}_{2 \text { peak }}, \mathrm{AT} \text {, peak power, SBP, DBP } \\
\mathrm{HR}_{\text {peak }}, \mathrm{HR}_{\text {rest }}, \mathrm{RER}\end{array}$ \\
\hline Eser 2020, Switzerland & MI & $\begin{array}{c}\text { HIIT }(34,8 \%) \\
\operatorname{MICT}(35,3 \%), \text { NR }\end{array}$ & $\begin{array}{l}\text { HIIT }(53 \pm 12.59) \\
\text { MICT }(59 \pm 7.41)\end{array}$ & SBP, DBP, HR peak, $\mathrm{HRR} 1 \mathrm{~min}$ \\
\hline Gao 2015, China & PCI & $\begin{array}{c}\text { HIIT }(18 \mathrm{M} / 4 \mathrm{~F}) \\
\mathrm{MICT}(16 \mathrm{M} / 5 \mathrm{~F}), \mathrm{ND}\end{array}$ & $\begin{array}{l}\text { HIIT }(59.4 \pm 7.9) \\
\text { MICT }(61.2 \pm 8)\end{array}$ & VO2peak, AT, peak power, LVEF \\
\hline $\begin{array}{l}\text { Ghardashi-Afousi 2018, } \\
\text { Iran }\end{array}$ & CABG & $\begin{array}{l}\text { HIIT (14M, 22\%) } \\
\text { MICT (14M, 22\%) }\end{array}$ & $\begin{array}{l}\text { HIIT }(53.9 \pm 3.44) \\
\text { MICT }(54.1 \pm 4.02)\end{array}$ & $\begin{array}{l}\text { SBP, DBP, } H_{\text {peak }}, H_{\text {rest }} \text { LVEF, LVEDD, } \\
\text { LVEDV, LVESD, LVESV }\end{array}$ \\
\hline $\begin{array}{l}\text { Jaureguizar 2016, Spain } \\
\text { Jaureguiza 2019, Spain }\end{array}$ & IHD & $\begin{array}{c}\text { HIIT }(28 \mathrm{M} / 8 \mathrm{~F}, 8 \%) \\
\text { MICT }(33 \mathrm{M} / 3 \mathrm{~F}, 13 \%) \\
\text { HIIT }(50 \mathrm{M} / 7 \mathrm{~F}) \\
\text { MICT }(42 \mathrm{M} / 11 \mathrm{~F}), \mathrm{NR}\end{array}$ & $\begin{array}{l}\text { HIIT }(58 \pm 11) \\
\text { MICT }(58 \pm 11) \\
\text { HIIT }(57.6 \pm 9.8) \\
\text { MICT }(58.3 \pm 9.5)\end{array}$ & $\mathrm{VO}_{2 \text { peak }}, \mathrm{AT}$, peak power, $\mathrm{HR}_{\text {peak }}, \mathrm{RER}$ \\
\hline Keteyian 2014, US & CAD & $\begin{array}{l}\operatorname{HITT}(11 \mathrm{M} / 5 \mathrm{~F}, 29 \%) \\
\operatorname{MICT}(12 \mathrm{M} / 1 \mathrm{~F}, 28 \%)\end{array}$ & $\begin{array}{c}\operatorname{HITT}(60 \pm 7) \\
\operatorname{MICT}(58 \pm 9)\end{array}$ & $\begin{array}{c}\mathrm{VO}_{2 \text { peak }}, \mathrm{AT}, \mathrm{SBP}, \mathrm{DBP}, \mathrm{HR}_{\text {rest }}, \mathrm{HR}_{\text {peak }} \\
\mathrm{HRR} 1 \mathrm{~min}, \mathrm{RER}, \mathrm{VE} / \mathrm{VCO}_{2} \text {, peak } \\
\mathrm{O}_{2} \text { pulse }\end{array}$ \\
\hline Kim 2015, South Korea & AMI & $\begin{array}{l}\text { HIIT(12M/2F, 13\%) } \\
\text { MICT(10M/4F, 13\%) }\end{array}$ & $\begin{array}{c}\operatorname{HIIT}(57 \pm 11.58) \\
\operatorname{MICT}(60.2 \pm 13.64)\end{array}$ & $\begin{array}{c}\mathrm{VO}_{2 \text { peak }}, \mathrm{HR}_{\text {peak }}, \mathrm{HR}_{\text {rest }}, \mathrm{HRR} 1 \mathrm{~min}, \\
\text { RER, LDL, HDL, TG }\end{array}$ \\
\hline Moholdt 2009, Norway & CABG & $\begin{array}{l}\operatorname{HIIT}(24 \mathrm{M} / 4 \mathrm{~F}, 15 \%) \\
\operatorname{MICT}(24 \mathrm{M} / 7 \mathrm{~F}, 11 \%)\end{array}$ & $\begin{array}{l}\operatorname{HIIT}(60.2 \pm 6.9) \\
\operatorname{MICT}(62 \pm 7.6)\end{array}$ & $\begin{array}{c}\mathrm{VO}_{2 \text { peak }}, \text { RER, HRR1min, } \mathrm{HR}_{\text {rest }}, \mathrm{QoL}, \\
\text { HDL-C, LDL-C, TG, LVEDV, LVESV, } \\
\text { LVEF, FBG }\end{array}$ \\
\hline Moholdt 2012, Norway & MI & $\begin{array}{c}\operatorname{HIIT}(25 \mathrm{M} / 5 \mathrm{~F}, 18 \%) \\
\operatorname{MICT}(49 \mathrm{M} / 10 \mathrm{~F}, 14 \%)\end{array}$ & $\begin{array}{l}\operatorname{HITT}(56.76 \pm 10.4) \\
\operatorname{MICT}(57.7 \pm 69.3)\end{array}$ & $\begin{array}{c}\mathrm{VO}_{2 \text { peak }}, \mathrm{HR}_{\text {peak }}, \mathrm{HR}_{\text {rest }}, \mathrm{RER}, \mathrm{HDL}-\mathrm{C} \text {, } \\
\mathrm{TG}, \mathrm{FBG}\end{array}$ \\
\hline Reed 2021, Canada & CAD & $\begin{array}{l}\text { HIIT(36M/7F, 12\%) } \\
\text { MICT(38M/6F, 18\%) }\end{array}$ & $\begin{array}{c}\text { HIIT(61 } \pm 7) \\
\operatorname{MICT}(60 \pm 7)\end{array}$ & SBP, DBP, QoL \\
\hline
\end{tabular}


Table 1. Cont.

\begin{tabular}{|c|c|c|c|c|}
\hline Study & Disease & Subjects $(N, \mathrm{DR})$ & Ages $(M \pm S D)$ & Outcomes \\
\hline Prado 2016, Brazil & CAD & $\begin{array}{c}\operatorname{HIIT}(14 \mathrm{M} / 3 \mathrm{~F}) \\
\text { MICT }(14 \mathrm{M} / 4 \mathrm{~F}), \mathrm{NR}\end{array}$ & $\begin{array}{l}\text { HIIT }(56.5 \pm 2.7) \\
\text { MICT }(61.3 \pm 2.2)\end{array}$ & $\begin{array}{c}\mathrm{VO}_{2 \text { peak }}, \mathrm{AT}, \mathrm{RER}, \mathrm{HR}_{\text {peak }}, \mathrm{OUES}, \\
\mathrm{VE} / \mathrm{VCO}_{2}\end{array}$ \\
\hline Taylor 2020, Australia & CAD & $\begin{array}{l}\text { HIIT (39M/7F, 4\%) } \\
\text { MICT (39M/8F, 9\%) }\end{array}$ & $\begin{array}{r}\operatorname{HIIT}(65 \pm 7) \\
\operatorname{MICT}(65 \pm 8)\end{array}$ & $\begin{array}{l}\mathrm{VO}_{2 \text { peak }}, \mathrm{HR}_{\text {peak }}, \mathrm{HR}_{\text {rest }}, \mathrm{RER} \text {, peak } \mathrm{O}_{2} \\
\text { pulse, OUES, LDL-C, HDL-C, TG, TC, } \\
\text { SBP, DBP, QoL, FBG }\end{array}$ \\
\hline Trachsel 2019, Canada & ACS & $\begin{array}{l}\operatorname{HIIT}(15 \mathrm{M} / 8 \mathrm{~F}, 38 \%) \\
\operatorname{MICT}(15 \mathrm{M} / 3 \mathrm{~F}, 5 \%)\end{array}$ & $\begin{array}{c}\text { HIIT }(63.6 \pm 9) \\
\text { MICT }(59.2 \pm 9.7)\end{array}$ & $\begin{array}{l}\mathrm{VO}_{2 \text { peak }}, \mathrm{OUES}, \mathrm{VE} / \mathrm{VCO}_{2}, \text { peak } \mathrm{O}_{2} \\
\text { pulse, peak power, } \mathrm{RER}, \mathrm{HR}_{\text {peak }}, \mathrm{HRR}\end{array}$ \\
\hline Warburton 2005, Canada & CAD & $\begin{array}{l}\operatorname{HIIT}(7 \mathrm{M}) \\
\operatorname{MICT}(7 \mathrm{M}), \mathrm{NR}\end{array}$ & $\begin{array}{c}\operatorname{HITT}(55 \pm 7) \\
\operatorname{MICT}(57 \pm 8)\end{array}$ & $\mathrm{HR}_{\text {peak }}$, peak $\mathrm{O}_{2}$ pulse, $\mathrm{VE} / \mathrm{VCO}_{2}, \mathrm{AT}$ \\
\hline Ye 2020, China & $\begin{array}{l}\text { Stroke }+ \\
\text { CAD }\end{array}$ & $\begin{array}{c}\text { HIIT }(43 \mathrm{M} / 17 \mathrm{~F}) \\
\text { MICT }(40 \mathrm{M} / 20 \mathrm{~F}), \mathrm{ND}\end{array}$ & $\begin{array}{l}\text { HIIT }(58.9 \pm 5.294) \\
\text { MICT }(59 \pm 4.643)\end{array}$ & $\mathrm{VO}_{2 \text { peak }}, \mathrm{AT}$, peak power, $\mathrm{LVEF}$ \\
\hline
\end{tabular}

$N$, number of patients; $\mathrm{M}$, male; F, female; DR, dropout rate; CAD, coronary artery disease; MI, myocardial infarction; CABG, coronary artery bypass grafting; IHD, ischemic heart disease; AMI, acute myocardial infarction; ACS, acute coronary syndrome; PCI, percutaneous transluminal coronary intervention; NR, dropout rate or gender not reported; ND, no dropout.

\subsection{Risk of Bias and Quality Assessment}

The Cochrane RoB Tool was used to analyze study quality. All studies were scored by two authors (LD and $\mathrm{KC})$ independently, and discrepancies were discussed and resolved. Seven studies were in low risk, seven studies were in moderate risk, and seven were in high risk (Figure 2). Egger's test was conducted for 16 outcomes (Table 2). No publication bias was found in all indicators except resting SBP $(p=0.006)$. We further performed the trim-and-fill method. The imputed studies produced a symmetrical funnel plot with an extra five studies filled (Figure 3).

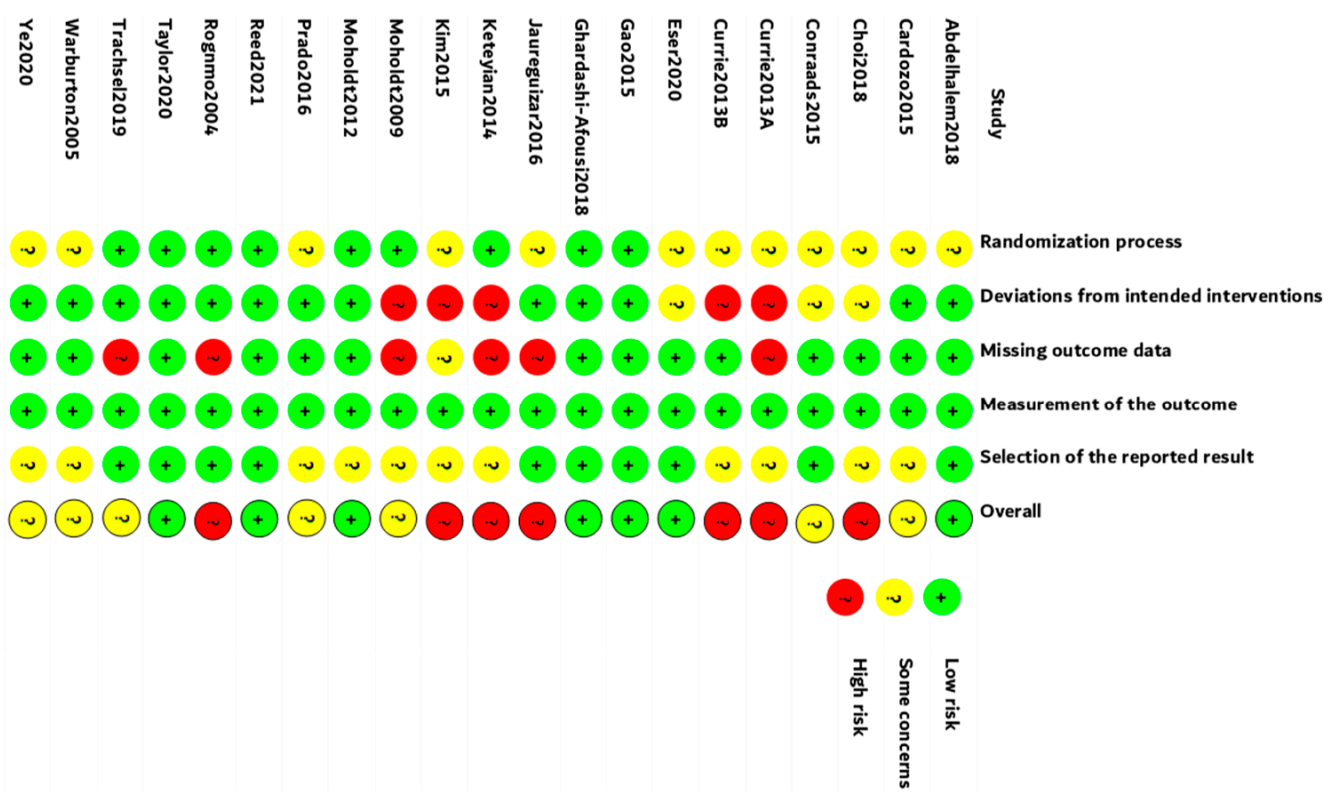

Figure 2. Quality analysis using Cochrane RoB Tool. 
Table 2. Egger's test of the included studies.

\begin{tabular}{|c|c|c|c|c|c|c|}
\hline Outcomes & $N$ & Std.Err & $\mathbf{t}$ & $p>|\mathrm{t}|$ & $95 \% \mathrm{CI}$ & Interval \\
\hline $\mathrm{VO}_{2 \text { peak }}$ & 16 & 0.367 & -1.20 & 0.252 & -1.225 & 0.348 \\
\hline AT & 9 & 0.441 & 1.41 & 0.197 & -0.396 & 1.640 \\
\hline $\mathrm{VE} / \mathrm{VCO}_{2}$ & 6 & 0.792 & 0.27 & 0.802 & -1.986 & 2.411 \\
\hline OUES & 5 & 1.526 & 0.19 & 0.864 & -4.572 & 5.141 \\
\hline peak $\mathrm{O}_{2}$ pulse & 6 & 1.113 & 0.08 & 0.943 & -3.006 & 3.176 \\
\hline LVEF & 5 & 1.253 & 2.95 & 0.060 & -0.295 & 7.677 \\
\hline peak power & 7 & 0.524 & -0.92 & 0.400 & -1.831 & 0.865 \\
\hline RER & 13 & 0.458 & -1.26 & 0.233 & -1.586 & 0.430 \\
\hline $\mathrm{HR}_{\text {peak }}$ & 15 & 0.461 & 0.07 & 0.946 & -0.964 & 1.028 \\
\hline$H_{\text {rest }}$ & 11 & 0.630 & 0.59 & 0.567 & -1.051 & 1.799 \\
\hline HRR1min & 7 & 2.285 & -0.06 & 0.956 & -6.008 & 5.741 \\
\hline SBP & 10 & 0.465 & 3.67 & 0.006 & 0.635 & 2.779 \\
\hline DBP & 10 & 0.876 & 1.19 & 0.268 & -0.977 & 3.061 \\
\hline HDL-C & 6 & 1.465 & 1.64 & 0.177 & -1.669 & 6.467 \\
\hline LDL-C & 5 & 1.360 & -1.15 & 0.333 & -5.893 & 2.763 \\
\hline TG & 6 & 0.935 & -0.89 & 0.423 & -3.431 & 1.764 \\
\hline
\end{tabular}

$\bar{N}$, number of analysed trails; Std.Err, standard error; $t, t$ value; $p>|t|$, probability values for publication bias examined by Egger's test.

Filled funnel plot with pseudo $95 \%$ confidence limits

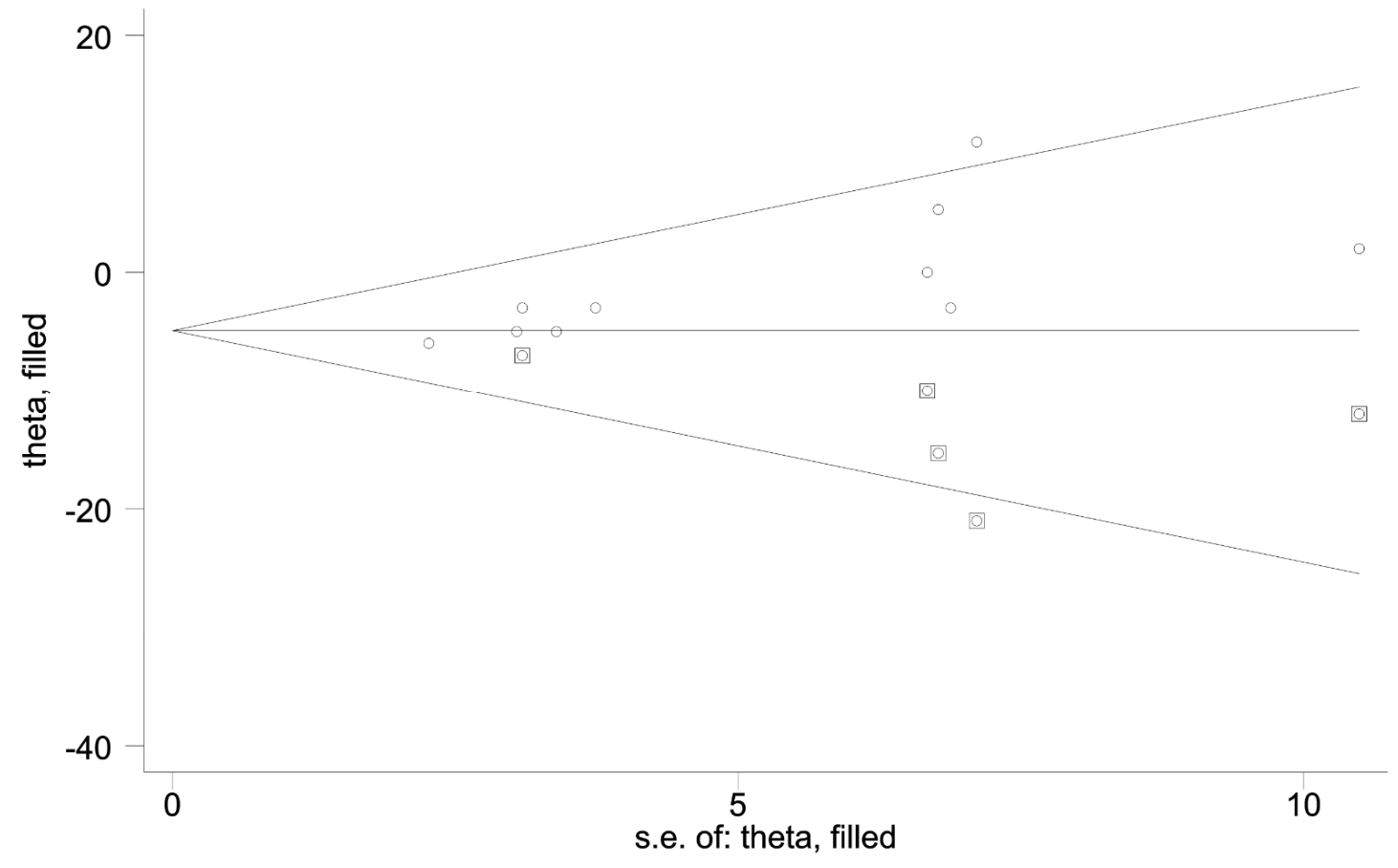

Figure 3. Funnel plot with trim and fill for the effect size of SBP. ( $\bigcirc$ :previous studies; @: filled studies).

\subsection{Effect of HIIT and MICT on $V O_{2 p e a k}$ in $C A D$ Patients}

$V O_{2 \text { peak }}$. The results of $\mathrm{VO}_{2 \text { peak }}$ were shown in Figure 4. Sixteen studies reported $\mathrm{VO}_{2 \text { peak }}$ as outcome $[22,25,27,28,30,32,33,35-38,41-44,46]$. The meta-analysis showed that both HIIT and MICT resulted in a significant increase in $\mathrm{VO}_{2 \text { peak }}(4.52 \mathrm{~mL} / \mathrm{kg} / \mathrm{min}, 95 \% \mathrm{CI}$ $[4.06,4.98], p<0.01$ and $2.36 \mathrm{~mL} / \mathrm{kg} / \mathrm{min}, 95 \% \mathrm{CI}[1.99,2.74], p<0.01$, respectively). Here, our data were mainly presented as the mean difference of the improvement magnitude of $\mathrm{VO}_{2 \text { peak }}$ between HIIT and MICT. We found that HIIT induces an overall significantly larger increase in $\mathrm{VO}_{2 \text { peak }}(1.92 \mathrm{~mL} / \mathrm{kg} / \mathrm{min}, 95 \% \mathrm{CI}[1.30,2.53], p<0.01)$ than MICT with low heterogeneity $\left(p=0.35, I^{2}=9 \%\right)$. As shown in Table S3, the subgroup analysis based on intervention duration ( $<12$ weeks, $\geq 12$ weeks) and training mode (treadmill, cycle 
ergometer, others) showed no significant subgroup difference (test for subgroup differences, $p=0.95, I^{2}=0 \% ; p=0.78, I^{2}=0 \%$, respectively). However, studies with medium and long HIIT intervals showed a significant greater increase in $\mathrm{VO}_{2 \text { peak }}(2.42 \mathrm{~mL} / \mathrm{kg} / \mathrm{min}, 95 \% \mathrm{CI}$ $[1.92,2.92], p<0.00001$ and $1.62 \mathrm{~mL} / \mathrm{kg} / \mathrm{min}, 95 \% \mathrm{CI}[0.49,2.75], p=0.005$, respectively) after HIIT, compared with MICT, while interventions with a short HIIT interval showed no difference $(0.31 \mathrm{~mL} / \mathrm{kg} / \mathrm{min}, 95 \% \mathrm{CI}[-1.25,1.87], p=0.70)$ after HIIT and MICT, and the test for the subgroup difference was significant $\left(p<0.05, I^{2}=72.5 \%\right)$. Similarly, studies with a work/rest ratio $>1$ in HIIT program showed a significantly greater improvement of $\mathrm{VO}_{2 \text { peak }}(2.30 \mathrm{~mL} / \mathrm{kg} / \mathrm{min}, 95 \% \mathrm{CI}[1.83,2.77], p<0.00001)$ than MICT, while interventions with a work/ rest ratio $\leq 1$ in HIIT program produced a similar effect $(0.84 \mathrm{~mL} / \mathrm{kg} / \mathrm{min}$, $95 \%$ CI $[-0.55,2.22], p=0.24)$ as MICT with a significant subgroup difference $(p=0.05$, $I^{2}=73.9 \%$ ). In addition, compared with isocaloric subgroup, studies using the nonisocaloric protocol showed an advantage in improvement of $\mathrm{VO}_{2 \text { peak }}(2.36 \mathrm{~mL} / \mathrm{kg} / \mathrm{min}$, $95 \%$ CI $[1.88,2.83], p<0.01)$ after HIIT than MICT. A subgroup analysis revealed significant subgroup difference $\left(p=0.01, I^{2}=84.1 \%\right)$. However, when Trachsel et al. [38] was removed, the heterogeneity dropped to $0 \%(p=0.52)$ from $10 \%(p=0.35)$ in the low work/rest ratio $(\leq 1)$ subgroup, with a trend favoring HIIT. Additionally, the significant difference between subgroups disappeared. In addition, when Trachsel et al. [38] was removed, the heterogeneity was similar $(0 \%)$ in the subgroup of a short HIIT interval $(\leq 1 \mathrm{~min})$, no significant subgroup difference was further observed $(p=0.12)$. However, the deletion of Trachsel et al. [38] elicited no effects on analysis. The study by Trachsel et al. [38] was of good quality but favored MICT in improving $\mathrm{VO}_{2 \text { peak }}$.

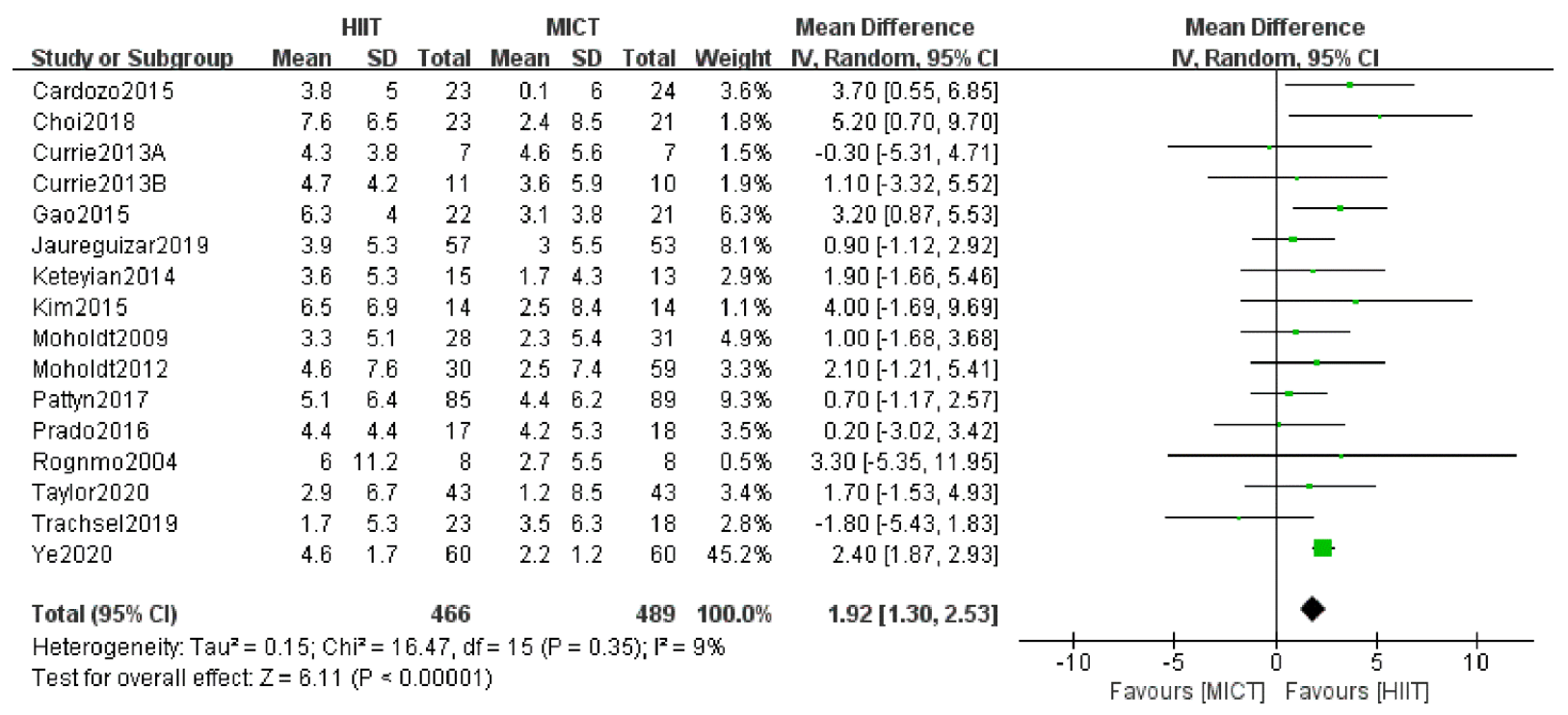

Figure 4. Changes in $\mathrm{VO}_{2 \text { peak }}$ between HIIT and MICT. HIIT, high-intensity interval training; MICT, moderate-intensity interval training. The green and black symbol means the mean difference of each studies and total mean difference.

\subsection{Effect of HIIT and MICT on Other Cardiorespiratory Parameters in CAD Patients}

AT. Ten studies $[27,28,30,34-37,39,43,44]$ reported AT as the outcome. The results showed both HIIT and MICT induced a significant improvement of AT $(2.63 \mathrm{~mL} / \mathrm{kg} / \mathrm{min}$, $95 \% \mathrm{CI}[1.59,3.67], p<0.01$ and $1.68 \mathrm{~mL} / \mathrm{kg} / \mathrm{min}, 95 \% \mathrm{CI}[0.69,2.68], p<0.01$, respectively). However, apparently HIIT resulted in a significant larger increase $(0.59 \mathrm{~mL} / \mathrm{kg} / \mathrm{min}, 95 \% \mathrm{CI}$ $[0.07,1.10], p<0.05$ ) in HIIT compared with MICT (Figure 5). The test for heterogeneity was low $\left(p=0.32, I^{2}=13 \%\right)$. 


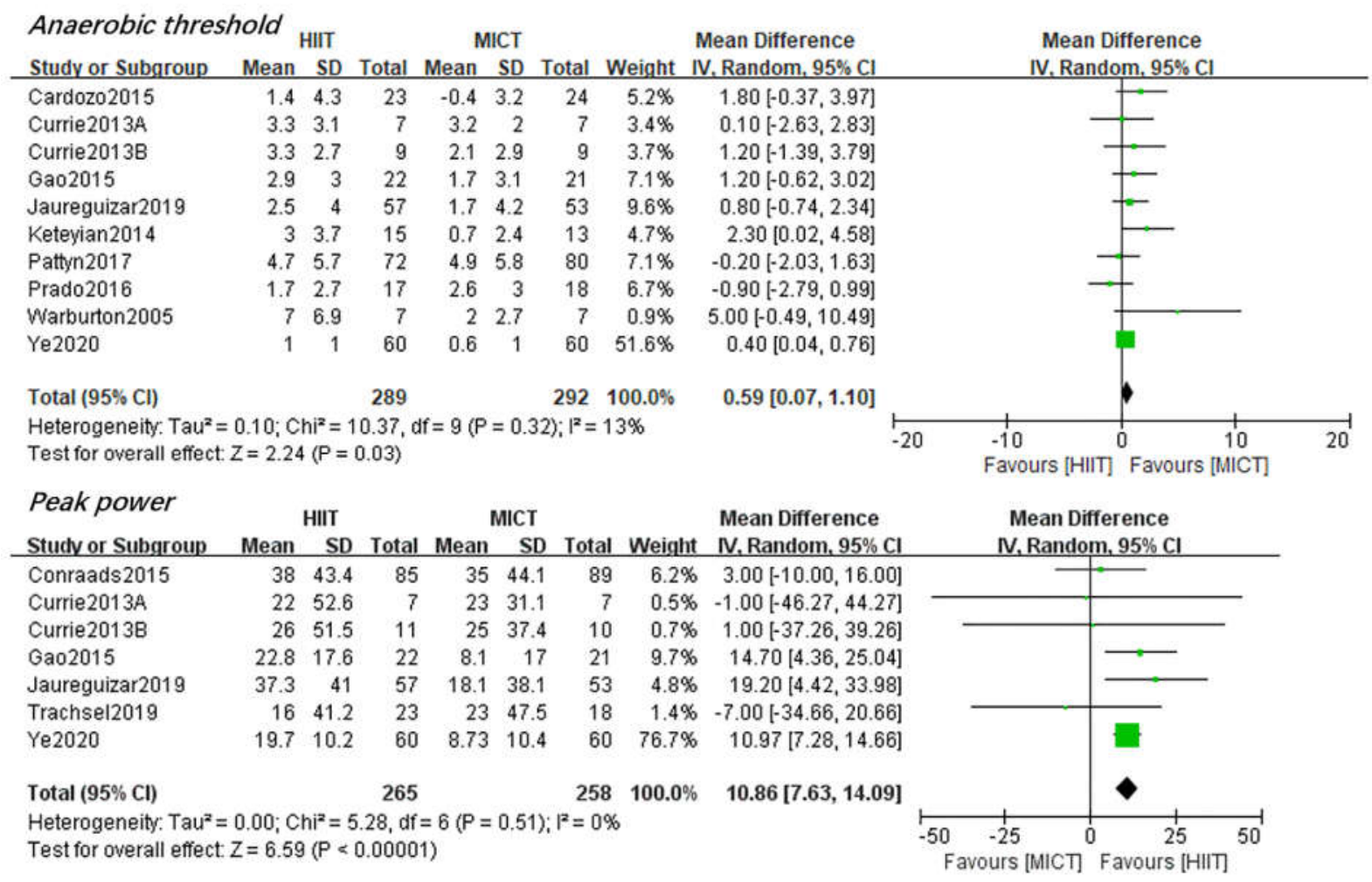

Figure 5. Changes in AT and Peak Power between HIIT and MICT. The green and black symbol means the mean difference of each studies and total mean difference.

Peak Power. Seven studies reported peak power as the outcome $[25,26,35,36,38,43,44]$. Additionally, both HIIT and MICT produced a significant increase in peak aerobic power (21.89 watt, 95\%CI [18.69, 25.08], $p<0.011$ and 11.30 watt, 95\%CI [8.06, 14.53], $p<0.01$, respectively).A significant larger improvement of peak power (10.86 watt, $95 \% \mathrm{CI}[7.63,14.09]$, $p<0.01$ ) after HIIT than MICT was observed (Figure 5). No heterogeneity was found $\left(p=0.51, I^{2}=0 \%\right)$.

HRR1min. The result of HRR1min led by seven studies $[26,28,31,32,37,38,42]$ showed significant changes after both HIIT and MICT intervention, however, no significant difference between HIIT and MICT $(-1.21 \mathrm{bpm}, 95 \% \mathrm{CI}[-3.87,1.45], p=0.37)$ was observed (Supplementary Figure S3). The heterogeneity between studies was statistically substantial $\left(p=0.01, I^{2}=62 \%\right)$. After removing Jaureguizar et al. [26] out of analysis, the advantage of HIIT became more obvious $(-2.55 \mathrm{bpm}, p=0.003)$ and the heterogeneity dropped to $0 \%$ $(p=0.83)$. The study led by Janureguizar et al. [26] strongly favored HIIT in lowering the heart rate in the first minute after peak exercise.

$H R_{\text {rest }}$. Eleven studies $[22,25,26,32,33,35-37,42,45,46]$ compared the effects of HIIT and MICT on the $\mathrm{HR}_{\text {rest }}$, and $\mathrm{HR}_{\text {rest }}$ was significantly reduced in both HIIT and MICT group $(-1.97 \mathrm{bpm}, 95 \% \mathrm{CI}[-3.39,-0.54], p<0.01$ and $-3.03 \mathrm{bpm}, 95 \% \mathrm{CI}[-4.39,-1.67]$, $p<0.01)$. The result also revealed no significant difference between groups $(-1.10 \mathrm{bpm}$, $95 \%$ CI $[-2.52,0.32], p=0.13$ ).

$H R_{\text {peak }}$. Fifteen studies $[22,25,27,30,31,33-39,42,45,46]$ evaluated the efficacy of HIIT and MICT on $\mathrm{HR}_{\text {peak }}$ and the $\mathrm{HR}_{\text {peak }}$ was significantly increased in HIIT and MICT $(1.67 \mathrm{bpm}, 95 \% \mathrm{CI}[0.29,3.05], p<0.05$ and $2.33 \mathrm{bpm}, 95 \% \mathrm{CI}[0.89,3.77], p<0.01)$. However, no significance was found between groups $(2.20 \mathrm{bpm}, 95 \% \mathrm{CI}[-0.47,4.88], p=0.11)$.

In addition, six studies $[27,28,34,37-39]$ reported the changes of VE/VCO2 slope after HIIT and MICT intervention; no significant changes were observed in both groups and a small effect towards MICT when compared with HIIT (SMD $-0.13,95 \%$ CI [ $-0.35,0.08]$, $p=0.23$ ). Another five studies $[27,28,34,38,46]$ evaluate the effects of HIIT and MICT on OUES, although both significantly improved OUES $(0.25,95 \%$ CI $[0.06,0.43], p<0.01$ vs. 
$0.18,95 \% \mathrm{CI}[0.02,0.34], p<0.05)$, an equal influence on OUES was found between HIIT and MICT (SMD 0.09 95\%CI [-0.12, 0.29], $p=0.40$ ). Additionally, six studies [25,34,37-39,46] compared the effects of HIIT and MICT on peak O2 pulse $(1.7,95 \% \mathrm{CI}[0.97,2.43], p<0.01$ and $1.37,95 \% \mathrm{CI}[0.67,2.07], p<0.01)$ and reported a small-to-mediate effect size favoring HIIT, but without significant group difference (SMD 0.34, 95\% CI $[-0.45,1.13], p=0.40$ ). Thirteen studies $[22,25,27,30,32-38,42,46]$ investigated the effects of HIIT on RER and compared with MICT and no effect was observed in both groups, also yielding an equal influence on RER (SMD 0.00, 95\%CI [-0.01, 0.01], $p=0.60$ ). The details can be found in Supplementary Materials.

\subsection{Effect of HIIT and MICT on CVD Risk Factors in CAD Patients}

Blood pressure. The results led by ten studies $[22,25,26,31,35-37,40,45,46]$ demonstrated that HIIT induced no significant changes in SBP $(0.84 \mathrm{mmHg}, 95 \% \mathrm{CI}[-1.53,3.21], p=0.49)$ and DBP $(0.67 \mathrm{mmHg}, 95 \% \mathrm{CI}[-1.77,3.11], p=0.59)$. Additionally, MICT induced no significant changes in SBP $(-1.86 \mathrm{mmHg}, 95 \% \mathrm{CI}[-4.18,0.46], p=0.12)$ and $\mathrm{DBP}(-1.25 \mathrm{mmHg}$, $95 \% \mathrm{CI}[-2.64,0.14], p=0.12)$.Interestingly, when comparing the effect induced by HIIT and $\mathrm{MICT}$, the pooled results favored MICT over HIIT in decreasing both SBP $(-3.61 \mathrm{mmHg}$, $95 \% \mathrm{CI}[-6.02,-1.20], p<0.01)$ and DBP $(-2.37 \mathrm{mmHg}, 95 \% \mathrm{CI}[-4.14,-0.60], p<0.01)$ without any heterogeneity (Figure 6).

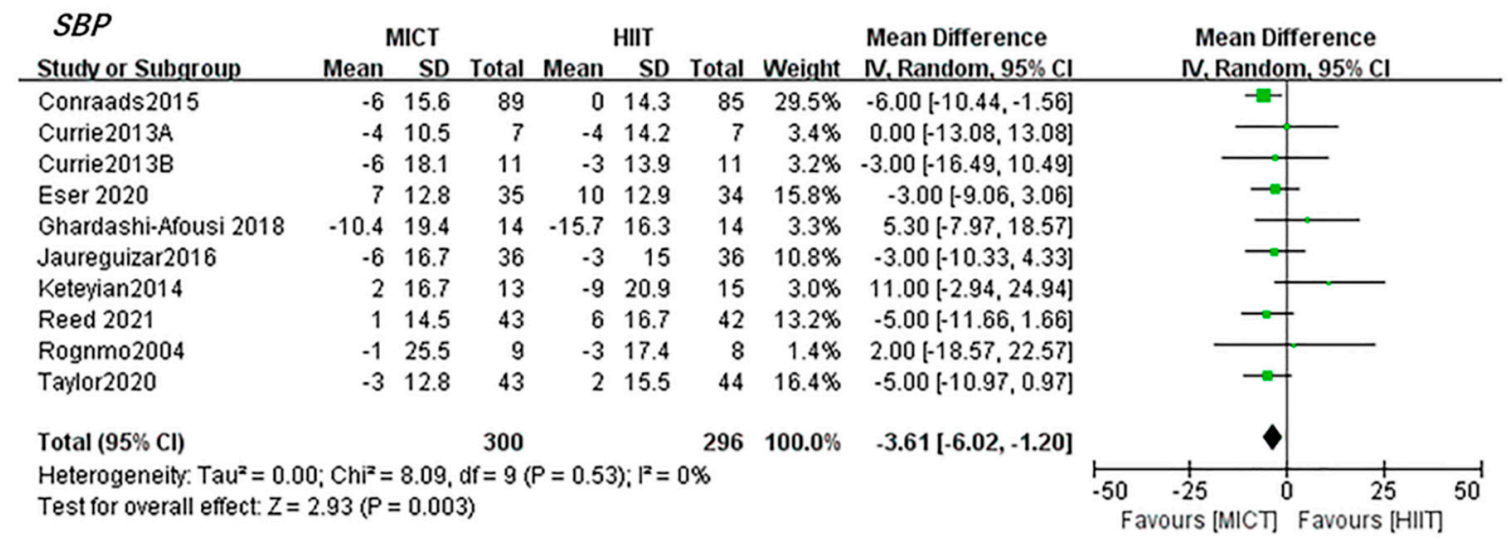

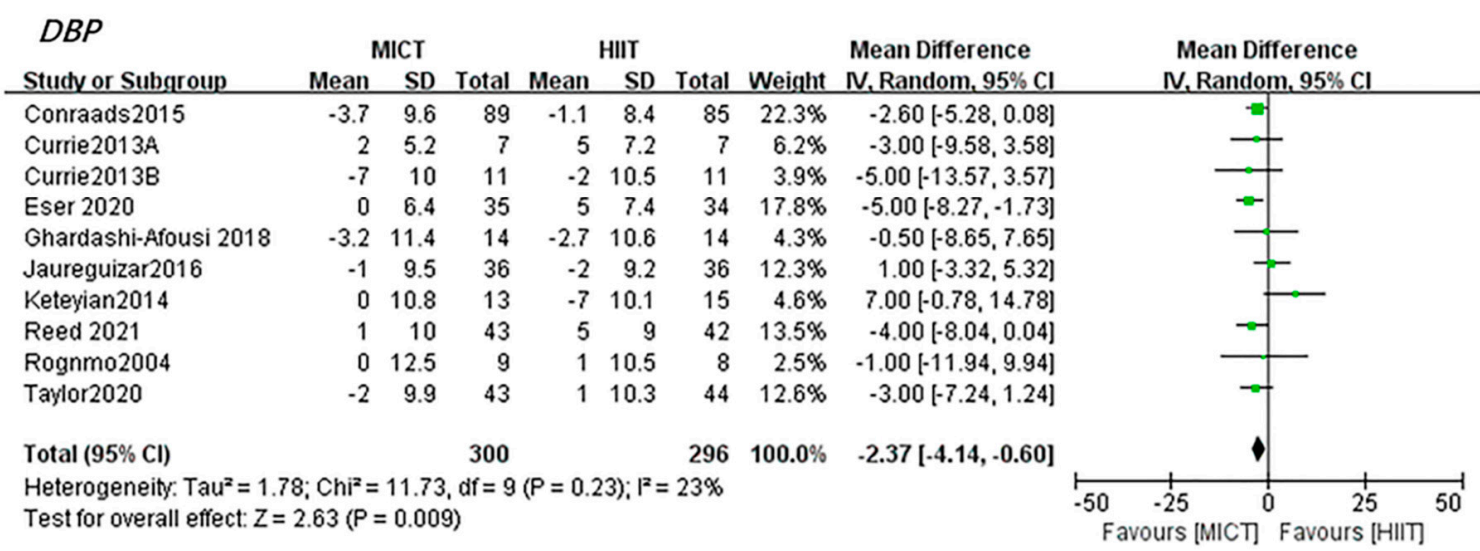

Figure 6. Changes in resting BP between HIIT and MICT. The green and black symbol means the mean difference of each studies and total mean difference.

Other parameters. HIIT significantly increased the level of HDL-C (SMD 0.31, 95\%CI [-0.01, 0.63], $p=0.05)$, while both HIIT and MICT did not significantly influence TG, TC, LDL-C and FBG. No significant difference was found in the improvement of HDL-C (SMD $0.14,95 \% \mathrm{CI}[-0.10,0.37], p=0.25)$, LDL-C (SMD $-0.10,95 \% \mathrm{CI}[-0.30,0.10], p=0.34)$, TG (SMD 0.00, 95\%CI $[-0.18,0.18], p=0.97)$, TC (SMD $-0.05,95 \% \mathrm{CI}[-0.28,0.17], p=0.66$ ), FBG (SMD $-0.01,95 \% \mathrm{CI}[-0.20,0.19], p=0.95$ ) between HIIT and MICT. The details can be found in Supplementary Materials. 


\subsection{Effect of HIIT and MICT on Left Ventricular Function in CAD Patients}

Five studies [32,43-45,47] compared the changes of LVEF after HIIT and MICT intervention and both reported significant improvement $(5.82 \%, 95 \% \mathrm{CI}[3.35,8.30], p<0.01$ and $1.78 \%, 95 \% \mathrm{CI}[0.25,3.32], p<0.05)$. However, no significant difference $(2.96 \%, 95 \% \mathrm{CI}$ $[-0.89,6.81], p=0.13)$ between groups was found. No significant difference was observed after HIIT and MICT in LVEDD, LVEDV, LVESV, LVESD and no significant was found between groups in LVEDD $(1.21 \mathrm{~mm}, 95 \% \mathrm{CI}[-1.82,4.24], p=0.43)$, LVEDV $(-2.06 \mathrm{~mL}, 95 \% \mathrm{CI}$ $[-9.14,5.02], p=0.57), \operatorname{LVESV}(-2.66 \mathrm{~mL}, 95 \% \mathrm{CI}[-6.35,1.03], p=0.16), \operatorname{LVESD}(-0.8 \mathrm{~mL}$, $95 \% \mathrm{CI}[-2.56,0.96], p=0.37)$. The details can be found in Supplementary Materials.

\subsection{Effect of HIIT and MICT on QoL in CAD Patients}

In total, five studies $[25,26,32,40,46]$ assessed the changes of QoL after HIIT and MICT intervention in this meta-analysis. There are two studies used the SF-36 form to evaluate the QoL [26,40] and one used SF-12 [25], a simplified version of SF-36, SMD was used to calculate the effect size. The results showed that both HIIT and MICT significantly increased the physical $(4.16,95 \% \mathrm{CI}[2.48,5.84], p<0.01$ and $4.41,95 \% \mathrm{CI}[2.72,6.10]$, $p<0.01)$ and mental component $(5.11,95 \% \mathrm{CI}[3.18,7.03], p<0.00001$ vs. $3.38,95 \% \mathrm{CI}$ $[1.67,5.09], p<0.01)$. However, no significant group difference in physical $(2.05,95 \% \mathrm{CI}$ $[-1.45,5.55], p=0.16)$ and mental $(2.05,95 \%$ CI $[-1.45,5.55], p=0.16)$ component was found. Additionally, in another three studies $[26,32,46]$ using the MacNew tool to evaluate QoL, both HIIT and MICT reported significant improvement in the emotional, physical, and social domain. However, no significant difference between HIIT and MICT in the emotional (SMD $0.18,95 \% \mathrm{CI}[-0.01,0.36], p=0.06)$, physical $(0.21,95 \% \mathrm{CI}[-0.02,0.44]$, $p=0.08)$ and social domain $(0.16,95 \% \mathrm{CI}[-0.08,0.39], p=0.19)$. The details can be found in Supplementary Materials.

\section{Discussion}

The aim of this systematic review was to explore the broad-spectrum health benefits of HIIT in CAD patients and compare with MICT. Additionally, the main findings were that HIIT has multiple positive effects on health-related fitness compared with MICT, resulting in larger improvement in $\mathrm{VO}_{2 \text { peak }}$, $\mathrm{AT}$ and peak power. Additionally, a subgroup analysis revealed that the medium and long HIIT intervals and higher HIIT work/rest ratio subgroups resulted in larger $\mathrm{VO}_{2 \text { peak }}$ improvement than short HIIT intervals and low HIIT work/rest ratio subgroup, respectively. In addition, the studies used non-isocaloric exercise protocol induced higher $\mathrm{VO}_{2 \text { peak }}$ gain than studies used isocaloric exercise protocol, indicating that the benefits of cardiorespiratory fitness might be determined by the total caloric consumption. MICT seems to be more effective in reducing resting SBP and DBP. However, HIIT and MICT equally affected other cardiorespiratory parameters, cardiovascular risk factors, QoL and left ventricular function.

$\mathrm{VO}_{2 \text { peak }}$ is an independent predictor of all-cause and cardiovascular-specific mortality [48]. Exercise intensity, rather than duration or frequency, is the most important variable in determining cardio protection and higher intensity exercise provides larger $\mathrm{VO}_{2 \text { peak }}$ changes [49]. HIIT can maximally stress the oxygen uptake and transportation as well as the utilization system, therefore providing the most effective stimulus for enhancing $\mathrm{VO}_{2 \text { peak }}$ [50]. Our finding showed that HIIT resulted in a larger gain of $1.92 \mathrm{~mL} / \mathrm{kg} / \mathrm{min}$ on $\mathrm{VO}_{2 \text { peak }}$ than $\mathrm{MICT}$, and this is in line with previous systematic reviews, which showed a larger $\mathrm{VO}_{2 \text { peak }}$ increase ranging from 1.25 to $1.78 \mathrm{~mL} / \mathrm{kg} / \mathrm{min}$ after HIIT versus MICT in CAD patients [16-19,51-53]. In addition, Hannan et al. [21] used SMD, instead of MD to account for the difference in measurement and reported a significant larger increase of $0.34 \mathrm{~mL} / \mathrm{kg} / \mathrm{min}$ by HIIT compared with MICT, which would be similar with our result if we had used SMD $(0.38 \mathrm{~mL} / \mathrm{kg} / \mathrm{min} ; 95 \% \mathrm{CI}[0.13,0.64], p=0.003)$ to calculate the effect size. Our result continued to support that HIIT is a promising alternative exercise protocol in the improvement of cardiorespiratory capacity in CAD patients. 
Our systematic review has included the most numbers of RCTs to date, enabling us to perform subgroup analysis. Our study found that both $<12$ and $\geq 12$ weeks durations favored HIIT and no significant subgroup difference was observed. This is consistent with a study led by Pattyn et al. [52] which did not distinguish CAD and heart failure patients. However, if we had conducted a subgroup analysis by $0-6$ weeks, $7-12$ weeks and $>12$ weeks duration as Hannan et al. [21], the result would similarly state that 7-12 weeks HIIT intervention elicited the largest SMD in cardiorespiratory fitness compared with $\leq 6$ weeks and $>12$ weeks duration in a mix of CAD patients, with or without heart failure. However, such a result should be interpreted with caution, as there are only three trials that have used an intervention duration of less than seven weeks [33,42,46] and only one trial used intervention longer than 12 weeks, which favored HIIT [34]. Thus, more studies with an intervention duration of $<7$ weeks and $>12$ weeks in CAD populations are needed in the future. Similarly, both treadmill and cycling exercise result in significantly larger $\mathrm{VO}_{2 \text { peak }}$ gain in HIIT versus MICT without significant subgroup difference. Our result is consistent with Pattyn et al. [52], which also reported no difference in $\mathrm{VO}_{2 \text { peak }}$ changes between different training modes. In fact, a previous meta-analysis comparing the treadmill and cycling exercise in $\mathrm{CR}$ showed a significant difference, However, the analysis was only performed to observe $\mathrm{VO}_{2 \text { peak }}$ changes pre and post MICT intervention, while HIIT was not explored [54]. Previous analysis in healthy people or athletes has concluded that HIIT with long intervals and higher work/rest ratio can produce larger benefits to cardiorespiratory fitness compared with MICT [50,55]. Additionally, consistent with these studies, our result also found larger $\mathrm{VO}_{2 \text { peak }}$ gain in HIIT in subgroups of mediate (1-3 $\mathrm{min}$ ) and long ( $\geq 4 \mathrm{~min}$ ) HIIT intervals, as well as higher work/rest ratio $(>1)$. In addition, total energy consumption of the intervention should be considered in the comparison of efficacy of health benefits between HIIT and MICT. An early study concluded that the increase in $\mathrm{VO}_{2 \text { peak }}$ by MICT is primarily determined by the total energy consumption [56]. Gevaert et al. [57] pointed out that it is the total volume of exercise that elevates the sufficient response to the exercise intervention. To eliminate this confounding factor, accumulating studies have used isocaloric exercise protocols; we found that when isocaloric exercise protocols were used, HIIT induced similar effects as MICT. This result was in accordance with previous systematic reviews in CAD patients [51], but different from studies in heart failure patients [58,59]. On the contrary, Pattyn et al. [52] reported superiority of isocaloric exercise protocols when simultaneously including CAD and heart failure patients together. Thus, a higher energy cost might be the underlying mechanism of larger $\mathrm{VO}_{2 \text { peak }}$ gain induced by HIIT. As the total energy expenditure of an exercise programme is determined by exercise frequency and duration, intensity, and programme length, the reasonable design of exercise-based CR should not focus on one single training characteristic (eg. intensity) but targeting at optimal total energy consumption, which was already acknowledged by a recent European Association of Preventive Cardiology position paper [60].

$\mathrm{AT}$ is the critical point of the transition from aerobic metabolism to anaerobic metabolism. Its increase enables $\mathrm{CAD}$ patients to perform aerobic exercise at a higher intensity, which will benefit their daily living activities. Our result suggested a significant $0.59 \mathrm{~mL} / \mathrm{kg} / \mathrm{min}$ larger improvement in oxygen uptake at AT in HIIT than MICT, which echoed the finding of Elliot et al. [16] and Xie et al. [19]. However, our results are inconsistent with Pattyn et al. [52], possibly due to the additional three studies with large samples that were included. The clinical role of other cardiopulmonary variables such as VE/VCO2 slope, OUES, peak $\mathrm{O}_{2}$ pulse and RER has also emerged as valuable, especially VE/VCO2 slope, which is an important independent prognostic marker in cardiac patients [61]. However, the benefit of HIIT and MICT on these factors is less investigated in previous meta-analysis. Our results suggested that $\mathrm{VE} / \mathrm{VCO}_{2}$ slope, OUES, peak $\mathrm{O}_{2}$ pulse and RER are equally affected by HIIT and MICT. This is in line with previous systematic reviews on CAD patients with or without reduced LVEF $[18,19,52]$. 
$\mathrm{HR}_{\text {rest }}$ is an indicator of autonomic nerve activity and elevated $\mathrm{HR}_{\text {rest }}$ is an established risk factor for cardiovascular events in patients with CAD. Elevated $\mathrm{HR}_{\text {rest }}$ is known to induce myocardial ischemia in CAD patients, while heart reduction is a recognized strategy to prevent ischemic episodes [62]. A dose-response of meta-analysis of prospective studies found an increased risk of coronary heart disease, sudden cardiac death, heart failure, atrial fibrillation, stroke, cardiovascular disease, total cancer and all-cause mortality with greater resting HR [63]. Liou et al. [17] reported a significantly larger $1.8 \mathrm{bpm}$ decrease in $\mathrm{HR}_{\text {rest }}$ after MICT compared with HIIT. On the contrary, our result showed no significant difference in $\mathrm{HR}_{\text {rest }}$ changes between the two interventions, which is in line with Pattyn et al. [52] and Qin et al. [53]. Regarding $\mathrm{HR}_{\text {peak, }}$ no statistical difference was observed between HIIT and MICT, which is in line with Qin [53]. While Pattyn et al. [52] found a significantly larger increase in $\mathrm{HR}_{\text {peak }}$ after HIIT compared with MICT in CAD patients, we speculate that the inconsistency may be due to the larger sample size we included. Nonetheless, higher peak HR reaching contributed to the increased peak oxygen uptake by increasing cardiac output. Overall, our results demonstrated that HIIT and MICT perform equally well in the adjusting HR; personalized features should be considered when making personalized prescriptions.

LVEF is regarded as a crucial cardiac function indicator. Pattyn et al. [52] and Qin et al. [53] both found no significant difference in LVEF\% gain between HIIT and MICT in CAD and HF patients, which was consistent with our result. Due to the limited number of studies and higher heterogeneity, this conclusion needs to be further determined in the future. We conducted the meta-analysis on other indicators of left ventricular function, such as LVEDD, LVEDV, LVESD and LVESV, which is, to our knowledge, the first meta-analysis comparing the effects of HIIT and MICT on these factors in CAD patients, although no significant difference was found between groups. Higher intensity tends to result in more intense stimulation to cardiac muscle, thus promoting a contraction. However, there few studies have investigated the effect of HIIT on stroke volume and cardiac output in CAD patients. Additionally, the findings in CAD patients with reduced LVEF revealed inconsistent results [64,65]. More studies are needed to further determine these effects in CAD in the future.

High blood pressure (BP) is a powerful predictor of cardiovascular morbidity and mortality. Lowering BP can decrease cardiovascular risk, with a $10-\mathrm{mmHg}$ reduction in SBP and is estimated to reduce all-cause mortality by $13 \%$ [66]. Surprisingly, no significant changes of SBP and DBP after HIIT and MICT intervention was found. This might be associated with the fact that most CAD patients included in this meta-analysis reported normal or not severe high blood pressure. However, our pooled results showed that when HIIT and MICT was compared, MICT seemed to induce a larger reduction in both SBP and DBP than HIIT. Additionally, these included trials did not give an exact explanation. Other systematic reviews all demonstrated no significant difference in BP changes between HIIT and MICT in CAD patients [19,52,53], except Elliot et al. [16] found an average decrease of $3.44 \mathrm{mmHg}(p=0.07)$ in SBP with a trend favoring MICT. In the present review, all ten studies had reported baseline medication status and most CAD patients were using medications for BP control, such as beta-blockers, calcium channel blockers, angiotensinconverting enzyme inhibitors, and nitrates. However, only six studies $[22,35-37,45,46]$ reported medication status during the exercise interventions, with medications changed in three studies $[35,37,46]$ and the rest of three $[22,36,45]$ remained unchanged. This would make it hard to interpret and discuss the underlying mechanism. However, there is no doubt that our result indicated a better effect of MICT than HIIT in reducing BP, which may help understand the potential benefits of MICT and enable the possibility of individual exercise prescriptions.

Blood lipids and glucose are also involved in the onset and development of CAD. Higher blood glucose is the hallmark of insulin resistance and type 2 diabetes, while exercise is the basic strategy to combat it [67]. Although previous studies have reported that HIIT significantly decreases fasting blood glucose or postprandial glucose $[68,69]$, 
systematic reviews have consistently reported a similar effect in lowing $\mathrm{HbA} 1 \mathrm{C}$ levels in Type 2 diabetes between HIIT and MICT [70]. The beneficial effects of HIIT on blood lipids is not consistent. No significant difference between HIIT and MICT in changes of HDL-C, LDL-C, TG, TC and FBG was observed in CAD patients, which is in line with the work of Xie et al. [19] and Pattyn et al. [52]. According to Pattyn et al. [52], this might be due to the pharmacological management of BP, cholesterol, and diabetes mellitus in the included patients, thus an additional effect of exercise training is therefore often absent or very small. In addition, all the included studies used short-term intervention, which might be not enough to induce positive adaption in blood risk indicators. In line with Gomes-Neto et al. [51], we found no difference in QoL changes in HIIT versus MICT, characterized by no significant difference in physical and mental components. Which means, either HIIT or MICT could have a positive impact on QoL, though no difference between the two interventions was observed. However, as it is limited by the number of studies, this result should be interpreted carefully.

In addition, we noticed that current $\mathrm{CR}$ guidelines recommend the inclusion of a standardized resistance-training program [71]. Palermi et al. [72] summarized the benefits of strength training to alleviate the burden of CVD and provide the rationale of adding strength training to the exercise prescription of people living with CVD. Additionally, a recent study led by Currie et al. [73] investigated the effect of HIIT combined with strength training on cardiovascular risk factors in CAD patients and revealed that resistance training was useful to improve the QoL and lipids. Additionally, it would be interesting to conduct more studies to explore the effect of strength training combined with HIIT or MICT on CAD patients in the future.

\section{Study Strength and Limitation}

This meta-analysis has included the greatest number of RCTs, including studies not previously included in other systematic reviews. All the trials have examined exercise intervention performed in a supervised manner rather than home-based. In addition, several studies performed by the same lab producing more than one publication was included only once for $\mathrm{VO}_{2 \text { peak }}$ analysis. Our review focused on CAD patients without reduced LVEF or heart failure. We investigated the effect of different intervention duration, HIIT intervals, HIIT work/ratio, training mode, energy consumption on $\mathrm{VO}_{2 \text { peak }}$ which has not been fully explored by previous reviews.

There are several limitations to our review. We included trials with a minimum four-week intervention in consideration of the consistency with previous relevant metaanalysis reviews. However, only two trials performed a four-week intervention while most of the included trials were eight weeks and above. Further systematic reviews could attempt to include all trials with a minimum of 8-week intervention in order to reduce the heterogeneity of exercise duration. In addition, most included studies had a small sample size and, more importantly, females only accounted for about one-fifth of the total sample size. This might cause bias to the results and make it more consistent with the intervention response characteristics of male patients, as they accounted for the vast majority of the sample. In addition, few trials have provided clear descriptions of the randomization and allocation process, which raises the possibility of performance bias. We did observe publication bias in the reporting of resting SBP evidenced by the Egger' test, although trim and fill was conducted later. Therefore, the corresponding result should be interpreted with caution. Furthermore, due to the high heterogeneity between the study protocol, a random-effects model was used, but the conclusion needs to be interpreted with caution. Finally, more studies need to be conducted to illustrate the adherence and relative safety risk of HIIT compared to traditional MICT and cover not only exercise-related adverse events, but a wider range of samples. 


\section{Conclusions}

This meta-analysis suggested that HIIT is superior to MICT in improving $\mathrm{VO}_{2 \text { peak, }}$, $\mathrm{VO}_{2}$ at $\mathrm{AT}$ and peak power in CAD patients. The optimal HIIT protocol in improving $\mathrm{VO}_{2 \text { peak }}$ might be those with mediate to longer intervals and higher work/rest ratios; it seemed that the efficacy of HIIT over MICT in improving $\mathrm{VO}_{2 \text { peak }}$ may not be influenced by intervention duration and training mode. In addition, the total energy consumption of exercise protocols determined the difference in $\mathrm{VO}_{2 \text { peak }}$ gain induced by HIIT and MICT, with the isocaloric protocol inducing similar effects. Both HIIT and MICT did not significantly influence resting $\mathrm{BP}$, however, MICT seemed to be more effective in reducing resting SBP and DBP than HIIT. HIIT and MICT equally significantly improved $\mathrm{HR}_{\text {rest, }}$ $\mathrm{HR}_{\text {peak }}$, HRR 1 min, OUES, LVEF\%, QoL, while had no significant influence on VE/ $\mathrm{VCO}_{2}$, peak $\mathrm{O}_{2}$ pulse, RER, and blood lipids. Further higher quality, large-sample, multicenter, long-term randomized interventional studies are needed to assess the effects of HIIT and MICT in CAD patients.

Supplementary Materials: The following are available online at https:/ /www.mdpi.com/article/ 10.3390/jcdd8110158/s1, Figures S1-S8: Changes in other parameters between HIIT and MICT, Table S1: Systematic literature search, Table S2: Intervention details. Table S3: Subgroup analyses of effects of HIIT versus MICT on $\mathrm{VO}_{2 \text { peak }}$.

Author Contributions: L.D. Data curation; Formal analysis; Visualization; Writing—Original draft preparation; Writing—Review and Editing, X.Z. Validation; Writing—Review and Editing; Funding acquisition; Visualization, K.C. Data curation; Formal analysis, X.R. Data curation; Formal analysis, S.C. Methodology; Project administration; Writing-Review and Editing; Supervision, Q.H. Conceptualization; Methodology; Writing — Review and Editing; Project administration; Funding acquisition. X.Z. contributed equally with L.D. to this review and could be considered as common first author. S.C. contributed equally with Q.H. to this review and could be considered as common corresponding author. All authors have read and agreed to the published version of the manuscript.

Funding: This research was funded by Ministry of Education of Humanities and Social Science Project (grant number 19YJCZH255) and the Fundamental Research Funds of Shandong University (grant number 2020GN064).

Data Availability Statement: Not applicable.

Conflicts of Interest: The authors have no potential conflict of interest regarding the research or publication of this manuscript.

\section{References}

1. Shengshou, H. Report on Cardiovascular Health and Diseases Burden in China: An Updated Summary of 2020. Chin. Circ. J. 2021, 36, 521-545.

2. Laukkanen, J.A.; Kurl, S.; Salonen, R.; Rauramaa, R.; Salonen, J.T. The predictive value of cardiorespiratory fitness for cardiovascular events in men with various risk profiles: A prospective population-based cohort study. Eur. Heart J. 2004, 25, 1428-1437. [CrossRef] [PubMed]

3. Myers, J.; Prakash, M.; Froelicher, V.; Do, D.; Partington, S.; Atwood, J.E. Exercise Capacity and Mortality among Men Referred for Exercise Testing. N. Engl. J. Med. 2002, 346, 793-801. [CrossRef] [PubMed]

4. Cornelissen, V.A.; Fagard, R.H. Effects of Endurance Training on Blood Pressure, Blood Pressure-Regulating Mechanisms, and Cardiovascular Risk Factors. Hypertension 2005, 46, 667-675. [CrossRef]

5. Oldridge, N. Exercise-based cardiac rehabilitation in patients with coronary heart disease: Meta-analysis outcomes revisited. Future Cardiol. 2012, 8, 729-751. [CrossRef]

6. Shepherd, C.W.; While, A.E. Cardiac rehabilitation and quality of life: A systematic review. Int. J. Nurs. Stud. 2012, 49, 755-771. [CrossRef]

7. Garber, C.E.; Blissmer, B.; Deschenes, M.R.; Franklin, B.A.; Lamonte, M.J.; Lee, I.-M.; Nieman, D.C.; Swain, D.P. American College of Sports Medicine position stand. Quantity and Quality of Exercise for Developing and Maintaining Cardiorespiratory, Musculoskeletal, and Neuromotor Fitness in Apparently Healthy Adults: Guidance for Prescribing Exercise. Med. Sci. Sports Exerc. 2011, 43, 1334-1359. [CrossRef]

8. Balady, G.J.; Chaitman, B.; Driscoll, D.; Foster, C.; Froelicher, E.; Gordon, N.; Pate, R.; Rippe, J.; Bazzarre, T. Recommendations for cardiovascular screening, staffing, and emergency policies at health/fitness facilities. Circulation 1998, 97, 2283-2293. [CrossRef] [PubMed] 
9. Fletcher, G.F.; Ades, P.A.; Kligfield, P.; Arena, R.; Balady, G.J.; Bittner, V.A.; Coke, L.A.; Fleg, J.L.; Forman, D.E.; Gerber, T.C.; et al. Exercise standards for testing and training: A scientific statement from the American Heart Association. Circulation 2013, 128, 873-934. [CrossRef]

10. Arena, R.; Myers, J.; Forman, D.E.; Lavie, C.J.; Guazzi, M. Should high-intensity-aerobic interval training become the clinical standard in heart failure? Heart Fail. Rev. 2013, 18, 95-105. [CrossRef]

11. Vanhees, L.; Rauch, B.; Piepoli, M.; van Buuren, F.; Takken, T.; Börjesson, M.; Bjarnason-Wehrens, B.; Doherty, P.; Dugmore, D.; Halle, M.; et al. Importance of characteristics and modalities of physical activity and exercise in the management of cardiovascular health in individuals with cardiovascular disease (Part III). Eur. J. Prev. Cardiol. 2012, 19, 1333-1356. [CrossRef]

12. Thum, J.S.; Parsons, G.; Whittle, T.; Astorino, T.A. High-Intensity Interval Training Elicits Higher Enjoyment than Moderate Intensity Continuous Exercise. PLoS ONE 2017, 12, e0166299. [CrossRef]

13. Vella, C.A.; Taylor, K.; Drummer, D. High-intensity interval and moderate-intensity continuous training elicit similar enjoyment and adherence levels in overweight and obese adults. Eur. J. Sport Sci. 2017, 17, 1203-1211. [CrossRef]

14. Guiraud, T.; Nigam, A.; Gremeaux, V.; Meyer, P.; Juneau, M.; Bosquet, L. High-Intensity Interval Training in Cardiac Rehabilitation. Sports Med. 2012, 42, 587-605. [CrossRef]

15. Quindry, J.C.; Franklin, B.A.; Chapman, M.; Humphrey, R.; Mathis, S. Benefits and Risks of High-Intensity Interval Training in Patients with Coronary Artery Disease. Am. J. Cardiol. 2019, 123, 1370-1377. [CrossRef]

16. Elliott, A.D.; Rajopadhyaya, K.; Bentley, D.J.; Beltrame, J.F.; Aromataris, E.C. Interval Training Versus Continuous Exercise in Patients with Coronary Artery Disease: A Meta-Analysis. Heart Lung Circ. 2015, 24, 149-157. [CrossRef] [PubMed]

17. Liou, K.; Ho, S.; Fildes, J.; Ooi, S.-Y. High Intensity Interval versus Moderate Intensity Continuous Training in Patients with Coronary Artery Disease: A Meta-analysis of Physiological and Clinical Parameters. Heart Lung Circ. 2016, 25, 166-174. [CrossRef]

18. Pattyn, N.; Coeckelberghs, E.; Buys, R.; Cornelissen, V.A.; Vanhees, L. Aerobic Interval Training vs. Moderate Continuous Training in Coronary Artery Disease Patients: A Systematic Review and Meta-Analysis. Sports Med. 2014, 44, 687-700. [CrossRef] [PubMed]

19. Xie, B.; Yan, X.; Cai, X.; Li, J. Effects of High-Intensity Interval Training on Aerobic Capacity in Cardiac Patients: A Systematic Review with Meta-Analysis. BioMed Res. Int. 2017, 2017, 1-16. [CrossRef]

20. Page, M.J.; McKenzie, J.E.; Bossuyt, P.M.; Boutron, I.; Hoffmann, T.C.; Mulrow, C.D.; Shamseer, L.; Tetzlaff, J.M.; Akl, E.A.; Brennan, S.E.; et al. The PRISMA 2020 statement: An updated guideline for reporting systematic reviews. Syst. Rev. 2021, 10, 89. [CrossRef]

21. Hannan, A.L.; Hing, W.; Simas, V.; Climstein, M.; Coombes, J.S.; Jayasinghe, R.; Byrnes, J.; Furness, J. High-intensity interval training versus moderate-intensity continuous training within cardiac rehabilitation: A systematic review and meta-analysis Open Access J. Sports Med. 2018, 9, 1-17. [CrossRef]

22. Rognmo, Ø.; Hetland, E.; Helgerud, J.; Hoff, J.; Slørdahl, S.A. High intensity aerobic interval exercise is superior to moderate intensity exercise for increasing aerobic capacity in patients with coronary artery disease. Eur. J. Cardiovasc. Prev. Rehabil. 2004, 11, 216-222. [CrossRef]

23. Amundsen, B.H.; Rognmo, Ø.; Hatlen-Rebhan, G.; Slørdahl, S.A. High-intensity aerobic exercise improves diastolic function in coronary artery disease. Scand. Cardiovasc. J. 2008, 42, 110-117. [CrossRef]

24. A Rocco, E.A.; Prado, D.M.; Silva, A.G.; Lazzari, J.M.A.; Bortz, P.C.; Rocco, D.F.M.; Rosa, C.G.; Furlan, V. Effect of continuous and interval exercise training on the PETCO2 response during a graded exercise test in patients with coronary artery disease. Clinics (Sao Paulo) 2012, 67, 623-627. [CrossRef]

25. Conraads, V.M.; Pattyn, N.; De Maeyer, C.; Beckers, P.J.; Coeckelberghs, E.; Cornelissen, V.A.; Denollet, J.; Frederix, G.; Goetschalckx, K.; Hoymans, V.Y.; et al. Aerobic interval training and continuous training equally improve aerobic exercise capacity in patients with coronary artery disease: The SAINTEX-CAD study. Int. J. Cardiol. 2015, 179, 203-210. [CrossRef]

26. Jaureguizar, K.V.; Vicente-Campos, D.; Bautista, L.R.; de la Peña, C.H.; Gómez, M.J.; Rueda, M.J.; Fernández, M.I. Effect of High-Intensity Interval Versus Continuous Exercise Training on Functional Capacity and Quality of Life in Patients With Coronary Artery Disease: A Randomized Clinical Trial. J. Cardiopulm. Rehabil. Prev. 2016, 36, 96-105. [CrossRef]

27. Prado, D.M.; Rocco, E.A.; Silva, A.G.; Rocco, D.F.; Pacheco, M.T.; Silva, P.F.; Furlan, V. Effects of continuous vs interval exercise training on oxygen uptake efficiency slope in patients with coronary artery disease. Braz. J. Med. Biol. Res. 2016, 49, e4890. [CrossRef]

28. Pattyn, N.; Beckers, P.; Cornelissen, V.A.; Coeckelberghs, E.; De Maeyer, C.; Frederix, G.; Goetschalckx, K.; Possemiers, N.; Schepers, D.; Van Craenenbroeck, E.M.; et al. The effect of aerobic interval training and continuous training on exercise capacity and its determinants. Acta Cardiol. 2017, 72, 328-340. [CrossRef]

29. Van De Heyning, C.M.; De Maeyer, C.; Pattyn, N.; Beckers, P.J.; Cornelissen, V.A.; Goetschalckx, K.; Possemiers, N.; Van Craenenbroeck, E.M.; Voigt, J.-U.; Vanhees, L.; et al. Impact of aerobic interval training and continuous training on left ventricular geometry and function: A SAINTEX-CAD substudy. Int. J. Cardiol. 2018, 257, 193-198. [CrossRef]

30. Villelabeitia-Jaureguizar, K.; Vicente-Campos, D.; Senen, A.B.; Jiménez, V.V.H.; Bautista, L.L.R.; Garrido-Lestache, M.E.B.; Chicharro, J.L. Mechanical efficiency of high versus moderate intensity aerobic exercise in coronary heart disease patients: A randomized clinical trial. Cardiol. J. 2019, 26, 130-137. [CrossRef]

31. Eser, P.; Jaeger, E.; Marcin, T.; Herzig, D.; Trachsel, L.; Wilhelm, M. Acute and chronic effects of high-intensity interval and moderate-intensity continuous exercise on heart rate and its variability after recent myocardial infarction: A randomized controlled trial. Ann. Phys. Rehabil. Med. 2020, 101444. [CrossRef] 
32. Moholdt, T.T.; Amundsen, B.H.; Rustad, L.A.; Wahba, A.; Løvø, K.T.; Gullikstad, L.R.; Bye, A.; Skogvoll, E.; Wisløff, U.; Slørdahl, S.A. Aerobic interval training versus continuous moderate exercise after coronary artery bypass surgery: A randomized study of cardiovascular effects and quality of life. Am. Heart J. 2009, 158, 1031-1037. [CrossRef]

33. Moholdt, T.; Aamot, I.L.; Granøien, I.; Gjerde, L.; Myklebust, G.; Walderhaug, L.; Brattbakk, L.; Hole, T.; Graven, T.; Stølen, T.O.; et al. Aerobic interval training increases peak oxygen uptake more than usual care exercise training in myocardial infarction patients: A randomized controlled study. Clin. Rehabil. 2011, 26, 33-44. [CrossRef]

34. Cardozo, G.G.; Oliveira, R.B.; Farinatti, P. Effects of High Intensity Interval versus Moderate Continuous Training on Markers of Ventilatory and Cardiac Efficiency in Coronary Heart Disease Patients. Sci. World J. 2015, 2015, 1-8. [CrossRef]

35. Currie, K.D.; Dubberley, J.B.; Mckelvie, R.S.; MacDonald, M.J. Low-Volume, High-Intensity Interval Training in Patients with CAD. Med. Sci. Sports Exerc. 2013, 45, 1436-1442. [CrossRef]

36. Currie, K.D.; Rosen, L.M.; Millar, P.J.; McKelvie, R.S.; MacDonald, M.J. Heart rate recovery and heart rate variability are unchanged in patients with coronary artery disease following 12 weeks of high-intensity interval and moderate-intensity endurance exercise training. Appl. Physiol. Nutr. Metab. 2013, 38, 644-650. [CrossRef]

37. Keteyian, S.J.; Hibner, B.A.; Bronsteen, K.; Kerrigan, D.; Aldred, H.A.; Reasons, L.M.; Saval, M.A.; Brawner, C.A.; Schairer, J.R.; Thompson, T.M.; et al. Greater Improvement in Cardiorespiratory Fitness Using Higher-Intensity Interval Training in the Standard Cardiac Rehabilitation Setting. J. Cardiopulm. Rehabil. Prev. 2014, 34, 98-105. [CrossRef]

38. Trachsel, L.-D.; Nigam, A.; Fortier, A.; Lalongé, J.; Juneau, M.; Gayda, M. Moderate-intensity continuous exercise is superior to high-intensity interval training in the proportion of VO2peak responders after ACS. Rev. Esp. Cardiol. (Engl. Ed.) 2019, 73, 725-733. [CrossRef]

39. Warburton, D.E.; McKenzie, D.C.; Haykowsky, M.J.; Taylor, A.; Shoemaker, P.; Ignaszewski, A.P.; Chan, S.Y. Effectiveness of High-Intensity Interval Training for the Rehabilitation of Patients With Coronary Artery Disease. Am. J. Cardiol. 2005, 95, 1080-1084. [CrossRef]

40. Reed, J.L.; Terada, T.; Cotie, L.M.; Tulloch, H.E.; Leenen, F.H.; Mistura, M.; Hans, H.; Wang, H.-W.; Vidal-Almela, S.; Reid, R.D.; et al. The effects of high-intensity interval training, Nordic walking and moderate-to-vigorous intensity continuous training on functional capacity, depression and quality of life in patients with coronary artery disease enrolled in cardiac rehabilitation: A randomized controlled trial (CRX study). Prog. Cardiovasc. Dis. 2021, in press. [CrossRef]

41. Choi, H.-Y.; Han, H.-J.; Choi, J.-W.; Jung, H.-Y.; Joa, K.-L. Superior Effects of High-Intensity Interval Training Compared to Conventional Therapy on Cardiovascular and Psychological Aspects in Myocardial Infarction. Ann. Rehabil. Med. 2018, 42, 145-153. [CrossRef]

42. Kim, C.; Choi, H.E.; Lim, M.H. Effect of High Interval Training in Acute Myocardial Infarction Patients with Drug-Eluting Stent. Am. J. Phys. Med. Rehabil. 2015, 94, 879-886. [CrossRef]

43. Gao, Z.; Ji, P.; Xia, Y.; Wang, L. Effects of different intensity aerobic exercise on cardiac function and exercise endurance in patients after percutaneous coronary intervention. Chin. J. Rehabil. Med. 2015, 30, 344-348.

44. Ye, R.; Sun, L.; Zhang, Q.; Wang, K. Influence of aerobic exercise intensity on cardiac function and exercise tolenrance in patients with stroke complicated coronaty heart disease. Chin. J. Cardiovasc. Rehabil. Med. 2020, 29, 536-540.

45. Ghardashi-Afousi, A.; Holisaz, M.T.; Shirvani, H.; Pishgoo, B. The effects of low-volume high-intensity interval versus moderate intensity continuous training on heart rate variability, and hemodynamic and echocardiography indices in men after coronary artery bypass grafting: A randomized clinical trial study. ARYA Atheroscler. 2018, 14, 260-271. [CrossRef]

46. Taylor, J.L.; Holland, D.J.; Keating, S.E.; Leveritt, M.D.; Gomersall, S.R.; Rowlands, A.V.; Bailey, T.G.; Coombes, J.S. Short-term and Long-term Feasibility, Safety, and Efficacy of High-Intensity Interval Training in Cardiac Rehabilitation. JAMA Cardiol. 2020, 5, 1382. [CrossRef]

47. Abdelhalem, A.; Shabana, A.; Onsy, A.; Gaafar, A. High intensity interval training exercise as a novel protocol for cardiac rehabilitation program in ischemic Egyptian patients with mild left ventricular dysfunction. Egypt. Heart J. 2018, 70, 287-294. [CrossRef]

48. Keteyian, S.J.; Brawner, C.A.; Savage, P.D.; Ehrman, J.K.; Schairer, J.; Divine, G.; Aldred, H.; Ophaug, K.; Ades, P.A. Peak aerobic capacity predicts prognosis in patients with coronary heart disease. Am. Heart J. 2008, 156, 292-300. [CrossRef]

49. Rankin, A.J.; MacIntyre, P.; Hillis, W.S.; Rankin, A.C. Walk or run? Is high-intensity exercise more effective than moderate-intensity exercise at reducing cardiovascular risk? Scott. Med. J. 2012, 57, 99-102. [CrossRef]

50. Buchheit, M.; Laursen, P.B. High-intensity interval training, solutions to the programming puzzle: Part I: Cardiopulmonary emphasis. Sports Med. 2013, 43, 313-338. [CrossRef]

51. Gomes-Neto, M.; Durães, A.R.; Dos Reis, H.F.C.; Neves, V.R.; Martinez, B.P.; Carvalho, V.O. High-intensity interval training versus moderate-intensity continuous training on exercise capacity and quality of life in patients with coronary artery disease: A systematic review and meta-analysis. Eur. J. Prev. Cardiol. 2017, 24, 1696-1707. [CrossRef]

52. Pattyn, N.; Beulque, R.; Cornelissen, V. Aerobic Interval vs. Continuous Training in Patients with Coronary Artery Disease or Heart Failure: An Updated Systematic Review and Meta-Analysis with a Focus on Secondary Outcomes. Sports Med. 2018, 48, 1189-1205. [CrossRef]

53. Qin, Y.; Bundhun, P.K.; Yuan, Z.-L.; Chen, M.-H. The effect of high-intensity interval training on exercise capacity in postmyocardial infarction patients: A systematic review and meta-analysis. Eur. J. Prev. Cardiol. 2021. [CrossRef]

54. Gerlach, S.; Mermier, C.; Kravitz, L.; Degnan, J.; Dalleck, L.; Zuhl, M. Comparison of Treadmill and Cycle Ergometer Exercise During Cardiac Rehabilitation: A Meta-analysis. Arch. Phys. Med. Rehabilitation 2020, 101, 690-699. [CrossRef] [PubMed] 
55. Cao, M.; Quan, M.; Zhuang, J. Effect of High-Intensity Interval Training versus Moderate-Intensity Continuous Training on Cardiorespiratory Fitness in Children and Adolescents: A Meta-Analysis. Int. J. Environ. Res. Public Health 2019, $16,1533$. [CrossRef] [PubMed]

56. Vromen, T.; Kraal, J.; Kuiper, J.; Spee, R.; Peek, N.; Kemps, H. The influence of training characteristics on the effect of aerobic exercise training in patients with chronic heart failure: A meta-regression analysis. Int. J. Cardiol. 2016, 208, 120-127. [CrossRef]

57. Gevaert, A.; Adams, V.; Bahls, M.; Bowen, T.S.; Cornelissen, V.; Dörr, M.; Hansen, D.; Mc Kemps, H.; Leeson, P.; Van Craenenbroeck, E.M.; et al. Towards a personalised approach in exercise-based cardiovascular rehabilitation: How can translational research help? A 'call to action' from the Section on Secondary Prevention and Cardiac Rehabilitation of the European Association of Preventive Cardiology. Eur. J. Prev. Cardiol. 2020, 27, 1369-1385. [CrossRef]

58. Araujo, B.; Leite, J.C.; Fuzari, H.; Pereira, D.S.R.; Remigio, M.I.; Dornelas, D.A.A.; Lima, C.S.; Cunha, B.D. Influence of HighIntensity Interval Training Versus Continuous Training on Functional Capacity in Individuals with Heart Failure: A Systematic Review and Meta-Analysis. J. Cardiopulm. Rehabil. Prev. 2019, 39, 293-298. [CrossRef] [PubMed]

59. Li, D.; Chen, P.; Zhu, J. The Effects of Interval Training and Continuous Training on Cardiopulmonary Fitness and Exercise Tolerance of Patients with Heart Failure-A Systematic Review and Meta-Analysis. Int. J. Environ. Res. Public Health 2021, 18, 6761. [CrossRef]

60. Hansen, D.; Abreu, A.; Ambrosetti, M.; Cornelissen, V.; Gevaert, A.; Kemps, H.; Laukkanen, J.A.; Pedretti, R.; Simonenko, M.; Wilhelm, M.; et al. Exercise intensity assessment and prescription in cardiovascular rehabilitation and beyond: Why and how: A position statement from the Secondary Prevention and Rehabilitation Section of the European Association of Preventive Cardiology. Eur. J. Prev. Cardiol. 2021. [CrossRef]

61. Sarullo, F.M.; Fazio, G.; Brusca, I.; Fasullo, S.; Paterna, S.; Licata, P.; Novo, G.; Novo, S.; Di Pasquale, P. Cardiopulmonary Exercise Testing in Patients with Chronic Heart Failure: Prognostic Comparison from Peak VO2 and VE/VCO2 Slope. Open Cardiovasc. Med. J. 2010, 4, 127-134. [CrossRef]

62. Ferrari, R.; Fox, K. Heart rate reduction in coronary artery disease and heart failure. Nat. Rev. Cardiol. 2016, 13, 493-501. [CrossRef]

63. Aune, D.; Sen, A.; Ó'Hartaigh, B.; Janszky, I.; Romundstad, P.R.; Tonstad, S.; Vatten, L.J. Resting heart rate and the risk of cardiovascular disease, total cancer, and all-cause mortality-A systematic review and dose-response meta-analysis of prospective studies. Nutr. Metab. Cardiovasc. Dis. 2017, 27, 504-517. [CrossRef]

64. Iellamo, F.; Manzi, V.; Caminiti, G.; Vitale, C.; Castagna, C.; Massaro, M.; Franchini, A.; Rosano, G.; Volterrani, M. Matched dose interval and continuous exercise training induce similar cardiorespiratory and metabolic adaptations in patients with heart failure. Int. J. Cardiol. 2013, 167, 2561-2565. [CrossRef] [PubMed]

65. Wisloff, U.; Stoylen, A.; Loennechen, J.P.; Bruvold, M.; Rognmo, O.; Haram, P.M.; Tjonna, A.E.; Helgerud, J.; Slordahl, S.A.; Lee, S.J.; et al. Superior cardiovascular effect of aerobic interval training versus moderate continuous training in heart failure patients: A randomized study. Circulation 2007, 115, 3086-3094. [CrossRef]

66. Ettehad, D.; Emdin, C.A.; Kiran, A.; Anderson, S.G.; Callender, T.; Emberson, J.; Chalmers, J.; Rodgers, A.; Rahimi, K. Blood pressure lowering for prevention of cardiovascular disease and death: A systematic review and meta-analysis. Lancet 2016, 387, 957-967. [CrossRef]

67. Palermi, S.; Iacono, O.; Sirico, F.; Modestino, M.; Ruosi, C.; Spera, R.; De Luca, M. The complex relationship between physical activity and diabetes: An overview. J. Basic Clin. Physiol. Pharmacol. 2021. [CrossRef]

68. Mendes, R.; Sousa, N.; Themudo-Barata, J.L.; Reis, V.M. High-Intensity Interval Training Versus Moderate-Intensity Continuous Training in Middle-Aged and Older Patients with Type 2 Diabetes: A Randomized Controlled Crossover Trial of the Acute Effects of Treadmill Walking on Glycemic Control. Int. J. Environ. Res. Public Health 2019, 16, 4163. [CrossRef]

69. Winding, K.M.; Munch, G.W.; Iepsen, U.W.; van Hall, G.; Pedersen, B.K.; Mortensen, S.P. The effect on glycaemic control of low-volume high-intensity interval training versus endurance training in individuals with type 2 diabetes. Diabetes Obes. Metab. 2018, 20, 1131-1139. [CrossRef]

70. De Nardi, A.T.; Tolves, T.; Lenzi, T.L.; Signori, L.U.; da Silva, A.M.V. High-intensity interval training versus continuous training on physiological and metabolic variables in prediabetes and type 2 diabetes: A meta-analysis. Diabetes Res. Clin. Pract. 2018, 137, 149-159. [CrossRef] [PubMed]

71. Balady, G.J.; Williams, M.A.; Ades, P.A.; Bittner, V.; Comoss, P.; Foody, J.A.; Franklin, B.; Sanderson, B.; Southard, D. Core components of cardiac rehabilitation/secondary prevention programs: 2007 update: A scientific statement from the American Heart Association Exercise, Cardiac Rehabilitation, and Prevention Committee, the Council on Clinical Cardiology; the Councils on Cardiovascular Nursing, Epidemiology and Prevention, and Nutrition, Physical Activity, and Metabolism; and the American Association of Cardiovascular and Pulmonary Rehabilitation. J. Cardiopulm. Rehabil. Prev. 2007, 27, 121-129. [PubMed]

72. Palermi, S.; Bragazzi, N.L.; Cular, D.; Ardigò, L.P.; Padulo, J. How chest press-based exercises can alleviate the burden of cardiovascular diseases. Hum. Mov. 2021, 22, 4. [CrossRef]

73. Currie, K.D.; Bailey, K.J.; Jung, M.E.; McKelvie, R.S.; MacDonald, M.J. Effects of resistance training combined with moderateintensity endurance or low-volume high-intensity interval exercise on cardiovascular risk factors in patients with coronary artery disease. J. Sci. Med. Sport 2015, 18, 637-642. [CrossRef] [PubMed] 